\title{
Optimization of Protocols for Pre-Embedding Immunogold Electron Microscopy of Neurons in Cell Cultures and Brains
}

Jung-Hwa Tao-Cheng ( $\sim$ chengs@ninds.nih.gov )

National Institutes of Health https://orcid.org/0000-0003-1897-5503

Virginia Crocker

National Institutes of Health

Sandra Lara Moreira

$\mathrm{NIH}$ : National Institutes of Health

Rita Azzam

$\mathrm{NIH}$ : National Institutes of Health

Methodology

Keywords: Synaptic proteins, electron microscopy

Posted Date: March 5th, 2021

DOI: https://doi.org/10.21203/rs.3.rs-268115/v1

License: (1) This work is licensed under a Creative Commons Attribution 4.0 International License. Read Full License 


\section{Abstract}

Immunogold labeling allows localization of proteins at the electron microscopy (EM) level of resolution, and quantification of signals. The present paper summarizes methodological issues and experiences gained from studies on the distribution of synaptic and other neuron-specific proteins in cell cultures and brain tissues via a pre-embedding method. An optimal protocol includes careful determination of a fixation condition for any particular antibody, a well-planned tissue processing procedure, and a strict evaluation of the credibility of the labeling. Here, tips and caveats on different steps of the sample preparation protocol are illustrated with examples. A good starting condition for EM-compatible fixation and permeabilization is $4 \%$ paraformaldehyde in PBS for $30 \mathrm{~min}$ at room temperature, followed by $30 \mathrm{~min}$ incubation with $0.1 \%$ saponin. An optimal condition can then be readjusted for each particular antibody. Each lot of the secondary antibody (conjugated with a $1.4 \mathrm{~nm}$ small gold particle) needs to be evaluated against known standards for labeling efficiency. Silver enhancement is required to make the small gold visible, and quality of the silver-enhanced signals can be affected by subsequent steps of osmium tetroxide treatment, uranyl acetate en bloc staining, and by detergent or ethanol used to clean the diamond knife for cutting thin sections. Most importantly, verification of signals requires understanding of the protein of interest in order to validate for correct localization of antibodies at expected epitopes on particular organelles, and quantification of signals needs to take into consideration the penetration gradient of reagents and clumping of secondary antibodies.

\section{Introduction}

Immunogold labeling of endogenous proteins with specific antibodies at the electron microscopy (EM) level offers high resolution in the localization of these proteins in intact cells [1, 2]. Information on the distribution and quantification of these proteins under different stimulation conditions can offer important clues to their functions $[3,4]$. The present paper summarizes this laboratory's over thirty years of experience in the pre-embedding technique of immunogold EM in dissociated rat hippocampal neuronal cultures and in perfusion-fixed mouse and rat brains, with particular focus on synaptic and other neuronal proteins.

For optimal structural preservation, samples processed for EM require a stronger fixation and a weaker permeabilization conditions than those processed for immunofluorescence light microscopy (LM). Thus, not all antibodies that work for immunofluorescence at the LM level will work for immunogold labeling at the EM level. The present paper will show examples of assessing optimal fixation and permeabilization conditions for each particular primary antibody.

The present paper will also illustrate the importance of evaluating the labeling efficiency of each lot of secondary antibody conjugated with a small $(1.4 \mathrm{~nm})$ gold particle [5, 6]. Silver or gold enhancement to enlarge the small gold particles into visible signals is another key step in the success of pre-embedding immunogold labeling $[7,8]$. Here, different enhancement reagents are compared to illustrate their specificity and efficiency. The enhanced signals can be affected by the concentration and composition of 
osmium tetroxide and uranyl acetate (UA) treatment during processing, and the silver particles in thin sections can deteriorate over time. The present paper lists examples of potential flaws and means to avoid them.

The present paper also lists several examples on interpretation of signals and means to verify the specificity of antibodies. We also list some common artifacts and cautions in quantifications of signals.

\section{Methods}

\section{Antibodies}

Mouse monoclonal antibodies (mAb) against a-CaMKII (clone 6G9(2), 1:100), GluR2 (clone 6C4, 1:100), and rabbit mAb against SynGAP (clone EPR 2883, 1:500) were from Millipore (Billerica, MA, USA); mouse mAb against IRSp53 (clone L117/1, undiluted), NR2B (clone N59/36, 1:100), pan Shank (clone N23B/49, which recognizes all three members of the Shank family: Shank 1, 2 and 3, 1:250), Shank 2 (clone N23B/6, 1:200), and Ip3 receptor (clone L24/18, 1:100) were from NeuroMab (Davis, CA, USA); rabbit polyclonal antibody (pAb) Shank3 (1:50) was from Santa Cruz (Dallas, TX, USA); rabbit pAb against synaptophysin (1:250) was from DAKO (Glostrup, Denmark); mouse mAb against synapsin I (clone 46.1, 1:250) and rabbit pAb against Homer 1, Homer 1b/c, Homer 2, Homer 1/2/3, and VGluT (all at 1:500) were from Synaptic Systems (Goettingen, Germany); mouse mAb against bassoon (clone SAP7F407, 1:100) was from Stressgen (Victoria, BC, Canada); rabbit pAb against NR2A/B (against C-terminus, 1:100) was from Chemicon (Temecula, CA, USA); mouse mAb against ryanodine receptor (clone 34C, at 1:500) was from Affinity BioReagents (Golden, CO, USA). Rabbit pAb raised to residues 290-307 of PSD-95 (at 1:500) was custom made by New England Peptide (Gardener, MA, USA).Mouse mAb against SV2 (1:500), was a gift from Dr. Erik S. Schweitzer; and rabbit pAb against chromogranin A (1:250) was a gift from Dr. Lee Eiden (NIMH, Bethesda, MD, USA).

Nanogold-Fab' secondary antibody (1.4 nm-sized gold covalently conjugated to the Fab' fragment of IgG), the HQ silver enhancement kit and the gold enhancement kit "Goldenhance EM" were from Nanoprobes (Yaphank, NY, USA); AURION R-Gent SE-EM, a silver enhancement kit from Aurion (Wageningen, the Netherlands) was distributed by Electron Microscopy Sciences (EMS, Hatfield, PA, USA).

\section{Fixation of dissociated rat hippocampal cultures}

Dissociated rat hippocampal neuronal cultures were grown as described [9]. Cultures were either grown on substrate-coated glass coverslips or on plastic tissue culture wares. The standard initial fixation in this lab for testing any new primary antibody is to use freshly diluted $4 \%$ paraformaldehyde (PF, $16 \%$ stock in sealed vials from EMS) in phosphate buffered saline (PBS) for $30 \mathrm{~min}$ at room temperature. Fixed samples were washed with PBS 4 times with 1 quick wash plus 3 times at 5 min interval each. Samples can then be stored in refrigerator at $4^{\circ} \mathrm{C}$ if need be. A storage time for up to one week yielded similar immunolabeling results for many antibodies. Depending on the initial assessment of the results, the concentration of PF and timing of the fixation can be adjusted. For some antibodies, a low concentration 
of glutaraldehyde $(0.05-0.2 \%)$ can be included to achieve better structural preservation. The optimal fixation conditions will have to be determined for each particular antibody (cf. supplementary information in $[10,11])$.

\section{Perfusion fixation of mouse and rat brains}

A good perfusion fixation $[1,12]$ will quickly bring fixative throughout the brain via the blood vessels, and the tissue will retain the fixative if the brain is kept intact and not cut up into thin slices or small pieces after the perfusion fixation. The standard procedure in this lab is to perfusion fix with $4 \%$ PF in PBS at room temperature, and to let the fixative stay in the brain not longer than 40 min to prevent over-fixation, which can lead to lower immunolabeling efficiency. Typically, the perfusion-fixed brain was dissected out and immersed in the same fixative until ready for vibratoming. The fixed brain was then transferred into PBS and vibratomed into $100 \mu \mathrm{m}$ thick slices in PBS. After 3 more washes in PBS at 5 min each, the brain slices are considered cleared of fixative and ready for immunolabeling. The brain slices can be stored in PBS in refrigerator at $4^{\circ} \mathrm{C}$ for up to 7-10 days without noticeable deterioration in structural preservation or labeling efficiency for most antibodies. The brain slices were then processed free-floating in 24-well tissue culture plates.

\section{Standard protocol of preembedding immunogold labeling}

The following steps are the standard procedure in this lab for initial testing with any new antibody:

1. Block and permeabilize thoroughly-washed samples with PBS containing $5 \%$ normal goat serum (NGS) and $0.1 \%$ saponin for $30 \mathrm{~min}$. Other reagents may be substituted for NGS to block non-specific labeling pending empirical results for each particular antibody.

2. Incubate with primary antibody made in PBS containing $5 \%$ NGS and $0.05 \%$ saponin for 1 hour at room temp. Carrying a control sample with no primary antibody incubation is strongly recommended.

3. Wash with PBS 4 times with 1 quick wash plus 3 times at 5 min interval each.

4. Incubate with secondary antibody (Nanogold-Fab' from Nanoprobes at 1:200) in PBS containing 5\% NGS and $0.05 \%$ Saponin for $1 \mathrm{hr}$ at room temp.

5. Wash with PBS 4 times with 1 quick wash plus 3 times at 5 min interval each.

6. Fix with $2 \%$ glutaraldehyde in PBS for 30 min at room temp, then store in fixative in refrigerator at $4^{\circ} \mathrm{C}$ if need be. A storage time for up to 4-6 weeks yielded similar immunolabeling results for many antibodies.

7. Wash with deionized water 5 times with 1 quick wash plus 4 times at 5 min interval each.

8. Silver enhance samples with the HQ kit from Nanoprobes in darkroom conditions under a red safety light. Free-floating brain slices were transferred into plastic baskets for easy handling and better control on solution changes under the safety light condition. Quickly mix equal volumes of the three reagents of the HQ silver enhancement kit and use immediately at room temperature. Optimal silver 
enhancement time should be determined for each antibody and for each lot of the HQ kit. Currently, a treatment time of $\sim 8-12$ min is the norm in our hands.

9. Wash samples with deionized water with 3 quick washes plus 5 times at 2 min each. After silver enhancement, samples typically showed a yellowish/brownish color visible by eye, and signals appeared as a dark brown reaction product under LM. The pattern of labeling under LM should be consistent with that of fluorescence images. Control sample with no primary antibody should not have visible signals and can serve as a quick verification of the validity of the immunolabeling procedure up to this step. On the other hand, if no or very little signal was detected from samples treated with primary antibody at this stage, samples can be further silver enhanced for additional time. We typically abandon samples if no signal is detected after a maximal of 15-20 min of silver enhancement. Experiments would be repeated at lower fixation conditions and/or higher concentrations of the primary antibody to increase labeling efficiency.

10. Change samples into $0.1 \mathrm{M}$ phosphate buffer at $\mathrm{pH} 7.4$ on ice and treat with fresh-made $0.2 \%$ osmium tetroxide [diluted from sealed vials of $4 \%$ aqueous stock (EMS)] in $0.1 \mathrm{M}$ phosphate buffer at $\mathrm{pH} 7.4$ for 30 minutes on ice.

11. Wash in $0.1 \mathrm{M}$ phosphate buffer with one quick wash and 2 times at $5 \mathrm{~min}$ each on ice, then either treat with steps 12-14 or proceed directly to dehydration with steps 15 onward.

12. Wash in $0.1 \mathrm{~N}$ acetate buffer at $\mathrm{pH} 5.0$ with one quick wash and 2 times at 5 min each on ice. ( $0.2 \mathrm{~N}$ acetate buffer stock was made with $0.2 \mathrm{~N}$ sodium acetate and $0.2 \mathrm{~N}$ acetic acid with a 3:1 volume ratio.)

13. Treat samples with $0.25 \%$ uranyl acetate (UA) in $0.1 \mathrm{~N}$ acetate buffer for $1 \mathrm{hr}$ on ice. The UA solution should be stored light-tight and cold at $4^{\circ} \mathrm{C}$.

14. Wash in $0.1 \mathrm{~N}$ acetate buffer thoroughly with 1 quick wash and 3 times at 5 min each on ice.

15. Dehydrate samples at room temperature in a graded series of ethanol at $50 \%, 70 \%, 90 \%$, and 3 changes of $100 \%$, and infiltrate in ethanol and epoxy resin mixtures at $1: 1$, and 1:2. It should be noted that cell culture samples benefit from a dehydration protocol with intervals shorter than the conventional 10 min interval for glutaraldehyde-fixed tissues. In this lab, immunogold-labeled monolayer samples were dehydrated at 4 min intervals, and perfusion-fixed brain slices were dehydrated at a 7 min schedule. This shortened dehydration schedule is helpful in preserving membrane structure for PF-fixed samples especially those that were fixed at a lower concentration for a shorter period of time. Time of infiltration with mixture of ethanol and epoxy resin varied between 15-30 min depending on the size of the sample. Monolayer samples were infiltrated between 15-20 min, and brain slices were infiltrated between 20-30 min.

16. Embed samples after 2 changes of $100 \%$ epoxy resin for $45-60$ min in $50^{\circ} \mathrm{C}$ oven, polymerize samples in the third change of resin at $50^{\circ} \mathrm{C}$ overnight, and at $60^{\circ} \mathrm{C}$ for two more days. Cell culture samples grown on tissue culture ware were embedded in the wells they were grown in, and cells grown on glass coverslips were embedded inverted, overhanging shallow embedding molds (Pelco DiscBlock embedding mold, Ted Pella, Redding, CA, USA) with the opening of the molds smaller than 
the size of the glass coverslips. Brain slices were either flat-embedded between two Permanox plastic slides (Thermo Fisher Scientific, Rochester, NY, USA), or with the interested area of the brain dissected out and oriented for cross-section in regular embedding molds.

\section{Thin sectioning and electron microscopy}

Epoxy-embedded cell culture samples were separated from the glass coverslips or the plastic tissue culture ware, and sections were typically cut en face. Flat embedded brain slices were trimmed for interested area of the brain and then oriented for either cross or en face sections.

400-mesh hexagonal copper grids were cleaned with acetone and air-dried before use, and ethanol should be avoided in cleaning grids because the residue left on the grids may interfere with silver-enhanced signals. The diamond knife used to cut thin sections should never be cleaned with detergent and should be rinsed and soaked with water overnight if it was cleaned with ethanol.

Thin sections at $~ 70 \mathrm{~nm}$ were stained with $1 \%$ UA for $15 \mathrm{~min}$ followed by $3 \%$ lead citrate for $5 \mathrm{~min}$ if the samples were not treated with UA en bloc during tissue processing. Samples that had UA en bloc staining can have sufficient contrast without the UA counterstaining of the thin sections. Sections were examined on a JEOL 1200EXII or a JEOL 200CX transmission EM and images were collected with a CCD digital camera system (XR-100 from AMT, Danvers, MA, USA).

\section{Results And Discussion}

Considerations and caveats at each step of the tissue processing protocol for pre-embedding immunogold labeling EM are listed here:

\section{Initial fixation affects structural preservation and immunolabeling efficiency}

Fixation typically decreases immunolabeling efficiency. In general, the classic EM fixative, glutaraldehyde, is not compatible with immunolabeling for the majority of antibodies, and paraformaldehyde (PF) is the preferred fixative for immunolabeling. Notably, structural preservation in PF-fixed samples without immunogold-labeled (Fig. 1a) is of better quality than those with labeling (Fig. 1c \& d) because the latter need to go through permeabilization treatment and extensive washing between the various incubation steps.

The quality of structural preservation of PF-fixed samples can be reasonably high if samples are optimally processed. Fig 1 a shows an example of dissociated hippocampal cultures fixed with $4 \%$ PF in PBS for $45 \mathrm{~min}$. The membranes and various organelles were similarly well-preserved when compared to a sample fixed with $4 \%$ glutaraldehyde in $0.1 \mathrm{M}$ cacodylate buffer (Fig. 1b). One conspicuous difference is that microtubules were only preserved in glutaraldehyde-fixed samples (arrows in Fig. 1b) but not in PFfixed samples (Fig. 1a). 
Typically, labeling efficiency is in reverse proportion to the degree of fixation. For some antibodies, the concentration of fixative and time of fixation need to be adjusted to a level where structural preservation will be further compromised. For example, structural preservation was better in the sample fixed at higher PF concentration and for a longer time (Fig. 1c) than the sample in Fig. 1d. However, even though membranes were not optimally preserved in this lesser-fixed sample (Fig. 1d), the resulting labeling at the postsynaptic compartment is still credible and quantification of labeling density is valid [13].

For perfusion-fixed brain tissues, over-fixation is a common cause for low labeling efficiency. Fig. 2a shows a brain sample that was perfusion-fixed with $4 \% \mathrm{PF}$ for $\sim 1$ hour before the brain was vibratomed into $100 \mu \mathrm{m}$ slices and washed in buffer. The labeling intensity with a shank 3 antibody at the postsynaptic density (PSD, arrows in Fig. 2a) was lower than that in another sample that was perfusionfixed for 40 minutes (Fig. 2 b).

\section{Permeabilization conditions affects membrane structure and labeling efficiency of plasma membrane proteins}

Immunolabeling reagents often need to pass the biological membranes to reach their target epitopes. A common reagent to make the membranes permeable is detergent, which modifies the membranes to different degrees depending on the type of detergent, and its concentration and treatment time. Based on our empirical experience with more than 200 different antibodies, the typical permeabilization condition we chose for the first try with any antibody is a $30 \mathrm{~min}$ incubation in $0.1 \%$ saponin at room temperature. With this treatment, the structure of the biological membrane was generally acceptably preserved even with a very mild fixation at 15 min of $4 \%$ PF.

Saponin works preferentially on cholesterol, which is more abundant in plasma membranes (PM) than in other biological membranes [15], and thus, may selectively alter the configuration of epitopes of PM proteins. In turn, labeling efficiency of antibodies against PM proteins may be affected by saponin treatment. For example, on PM of skeletal muscles, labeling efficiency of a glucose transporter (GluT4) was substantially affected by the concentration of saponin [16]. Here, we compared the labeling efficiency of PM labeling of a glutamate receptor (GluR2, an AMPA receptor of subtype 2) between samples permeabilized with saponin and samples treated with $10 \mathrm{~min}$ of $50 \%$ ethanol followed by several washes of water to clear ethanol off the tissue. For these ethanol-permeabilized samples, saponin was also omitted from all subsequent steps.

PM of neuronal soma was specifically labeled with GlurR2 [17] after both permeabilization treatments (Fig. 3). However, PM appeared wavier in saponin-treated samples (Fig. 3a, b) than in ethanol-treated samples (Fig. 3c, d), and the labeling density was $40-55 \%$ lower in saponin-treated samples than in ethanol-treated samples (Fig. 3e, Additional File 1).

In summary, antibodies that worked well for immuno-fluorescence light microscopy (LM) may not necessarily work for immunogold labeling by EM. The two key factors for EM are the additional fixation required for EM samples, and milder permeabilization conditions needed to preserve membrane 
structures in order to interpret EM images. Our recommended preliminary testing for any antibody is to carry LM experiments with an EM-compatible fixation and permeabilization conditions. For example, samples can be fixed at 4\% PF for 15, 30, 60 min initially and then permeabilized with $0.1 \%$ saponin for $30 \mathrm{~min}$ to see if fluorescence signals survived these conditions. If so, chances are good that the immunogold labeling would work for these antibodies at the EM level. Once it is established that a particular antibody works at the EM level, additional tweaking on fixation and permeabilization conditions can be tried to optimize between the balance of achieving better structural preservation and higher labeling efficiency.

It should be noted that Triton X, a popular detergent for immunofluorescence LM, should not be routinely used for EM studies because the biological membranes could be easily dissolved by this detergent and render the EM images uninterpretable. Triton $X$ can only be used under specialized conditions at very low concentration and in conjunction with additional EM fixatives [18].

\section{Quality control of secondary antibody}

After testing a few different types of secondary antibodies conjugated with gold, our choice reagent is Nanogold-Fab' from Nanoprobes (Yaphank, NY) due to its higher labeling efficiency [5] and stability over time. This secondary antibody is a Fab' fragment of the IgG, conjugated with a covalently-bound small gold $(1.4 \mathrm{~nm})$ [6]. Although the great majority of Nanogold secondary antibodies we received worked well, a quality check on any new shipment is still advised. One example is illustrated in Fig. 4 where sister cultures of hippocampal neurons were identically fixed and permeabilized, labeled with a CaMKII antibody, and subsequently incubated with two different lots of Nanogold secondary antibodies. Labeling density near the postsynaptic density (PSD) of one sample (Fig. $4 a$ ) is only $17 \%$ of the other (Fig. 4 b, C). A possible reason for the low efficiency in lot 1 may be low percentage of conjugation of the gold to the secondary antibodies.

We also carried out periodic quality checks on the Nanogold secondary antibodies in our stock, and found that Nanogold antibodies were very stable, even over many years, if stored properly in the refrigerator at $4^{\circ} \mathrm{C}$. Additional File 2 indicates that Nanogold antibodies which had been stored for more than 4 years still yielded similar labeling intensity (80-92\%) to recently shipped Nanogold.

Freezing Nanogold is the only handling condition in our hands that caused a substantial decrease in labeling efficiency. Thus, one reason for some lots of Nanogold to perform disappointingly (cf. Fig. 4a) could be poor handling en route, e. g. accidental freezing during shipment. The typical practice in this lab is to test any new lot of Nanogold secondary antibody with a reliable primary and secondary ab as a positive control. Once it passed the test to yield acceptable labeling efficiency, we would buy more of the same lot and keep them for several years. We always have several lots of secondary Nanogold on hand and do not wait until the supplies are very low to buy and test replacement.

\section{Comparison of labeling efficiency by different silver or gold enhancement reagents}


Since the gold particle conjugated to the secondary antibody is too small $(1.4 \mathrm{~nm})$ to be visible by conventional transmission EM, samples need to go through silver or gold enhancement to enlarge the small gold to become visible signals [7, 8]. The efficiency among three different types of silver or gold enhancement reagents are compared here.

The HQ silver enhancement kit from Nanoprobes needs to be handled in a darkroom under a red safety light. We found that it does not require absolute darkness and can be accommodated with a closed room with lights off, and a red safety light on the bench. The silver enhancement kit from Aurion, AURION RGent SE-EM, and the gold enhancement kit from Nanoprobes, Goldenhance, are used under regular bench conditions. However, even with the inconvenience of a darkened bench and the safety light requirement, the HQ kit turned out to be our choice reagent due to its higher efficiency and high specificity.

Figs. $5 \mathrm{a} \& \mathrm{~b}$ illustrate the difference between the $\mathrm{HQ}$ kit and the Aurion silver enhancement reagents in a parallel experiment. Hippocampal cultures labeled for SV2, a synaptic vesicle (SV) membrane protein [11], showed specific labeling on SVs in presynaptic terminals with both enhancement reagents. The Aurion enhancement kit yielded homogeneous-sized particles after $60 \mathrm{~min}$ of exposure at room temperature (Fig. $5 a)$, the image of which is perfectly suitable for qualitatively illustrating the specific labeling. The Aurion kit also has the convenience that one can monitor for the progression of reaction product under a light microscope without the guess work on the treatment time. On the other hand, the HQ silver enhancement kit produced heterogeneous-sized particle after 6 min of treatment at room temperature (Fig. $5 b$ ). This particular sample in Fig. 5b was clearly over-enhanced that the large and crowded silver particles obscured the underneath structure of the SVs. However, labeling intensity is much higher by the HQ kit at 2.7 - 3.8 folds over the Aurion kit (Fig. 5d, Additional File 3). This higher detection sensitivity can be especially advantageous when detecting antigens of low concentration. Notably, the silver enhancement process progresses with time [1] and is temperature-dependent [7]. Thus, the over-sized signals can be remedied by reducing time (Fig. 5c) or temperature of the silver enhancement treatment.

Labeling efficiency was also tested between the Nanoprobes HQ silver enhancement kit and the Nanoprobes Goldenhance reagents. Samples were identically treated except for the silver (Fig. 6a, c) or gold (Fig. 6b, d) enhancement steps for 8 min and 10 min each, respectively. The efficiency of the HQ kit is much higher by $2.3-4.1$ folds (Fig. 6e, Additional File 4).

One major drawback of the Nanoprobes Goldenhance kit in our hands is that there were nonspecific, fine particles all over membranous structures (Figs. $6 b, d, f)$. These artifacts are distinct from the real signals localized on the SVs (Fig. 6b), and were not caused by counterstaining (Fig. 6d). Fig. 6f shows a neuronal soma where membranes of different organelles, including Golgi, mitochondria and multivesicular body, were all decorated with such fine particles. One cautionary sign of the non-specificity of this artifact is that the particles in question are all over every piece of membrane, including the inner and outer membranes of the nuclear envelope and the inner and outer membranes of mitochondria where it is known that the compositions of these membranes are different $[19,20]$. Although there were studies that successfully used Goldenhance without such nonspecific artifacts [21], we could not easy get rid of these 
artifacts and decided to use HQ because of its higher labeling specificity and efficiency (Fig. 6e, Additional File 4).

\section{Recommendations on managing the HQ silver enhancement kit}

Each lot of HQ silver enhancement kit needs to be characterized to determine an optimal development time. Figs. 7a \& b show a pair of samples identically treated except for silver enhancement time. The sample enhanced for $5 \mathrm{~min}$ had smaller particles (Fig. 7a) than the sample enhanced for 8 min (Fig. 7b). It should be noted that these samples were processed 15 years ago, and we have noticed that the HQ kits in recent years required longer development time. Figs. 7c \& $d$ show a pair of parallel samples silver enhanced with two different lots of $\mathrm{HQ}$ kits for 12 min each. The sizes of the particles are smaller with one lot (Fig.7c) than the other lot (Fig. 7d). Thus, the silver enhancement time should be adjusted for different lots of $\mathrm{HQ}$ kits in order to achieve the desired size range of the particles.

Our typical practice was to buy several $\mathrm{HQ}$ kits of the same lot and store them at $-20^{\circ} \mathrm{C}$. We thawed the $\mathrm{HQ}$ reagent, one kit at a time, and refrigerate the unused portion at $4^{\circ} \mathrm{C}$ to be used within a month. If the thawed reagents were not expected to be used up within that time frame, we aliquoted the three components of the HQ kit into equal volumes and stored them frozen. The reagents were stable for approximately one year in our hands. Longer storage times either in the refrigerator or in the freezer, sometimes changed the optimized development time. We also noticed a trend that the quality of the silver particles tended to have more problems when the HQ reagents used were older.

\section{Caveats on osmium treatment of silver enhanced samples}

Osmium tetroxide treatment is generally required for EM samples in order to preserve membrane structures. However, it is also shown to reduce the size of the silver enhanced particles [8]. In earlier tries, an osmium tetroxide treatment at $1 \%$ sometimes yielded irregular-shaped particles with a fuzzy border (Fig. 8a). We also found that the silver enhanced particles are poorly preserved with "reduced osmium" treatment (Fig. 8b), where potassium ferrocyanide is included with osmium tetroxide.

In order to consistently produce sharp looking silver particles, the routine in our lab now is to use freshmade $0.2 \%$ osmium tetroxide in $0.1 \mathrm{M}$ phosphate buffer at $\mathrm{pH} 7.4$ for $30 \mathrm{~min}$ on ice. Because osmium tetroxide is very volatile, a $0.2 \%$ solution made ahead of time may become less in concentration by the time it is used. The consequence of which would be poorly preserved membranes.

One cautionary note is that on rare occasions, the osmium treatment step may bleach the silver enhancement product, resulting in samples with no silver particles at all. The fact that such samples had absolutely no silver particles anywhere in the samples raised concerns because even the control samples where primary antibody was omitted should still have some background particles. Thus, some chemical reactions must have bleached all signals between the steps of silver enhancement and the rest of the processing procedures. We now closely monitor the yellowish/brownish color of silver-enhanced samples by eye while adding the osmium tetroxide solution. We have found that if "bleaching" is to occur, the color 
would disappear within the first minute. It is not worth proceeding with the "bleached" samples because no signals will be preserved if bleaching occurred at this step. Also, to guard against the possibility that the silver enhancement reagents may contribute to this "bleaching" phenomenon, the repeat experiment would be silver enhanced with a different batch of the HQ kit.

\section{Caveats on uranyl acetate (UA) en bloc staining}

To increase contrast, tissues are often en bloc stained with UA following osmium treatment and prior to dehydration. This UA en bloc staining was carried out at cold temperature $\left(4^{\circ} \mathrm{C}\right)$ under light-tight conditions either wrapped in foil or kept in the refrigerator. Although this step is not necessary, we found the increased contrast in immunogold-labeled samples, which were typically weakly fixed with lesser structural preservation, worthy of this additional step.

Our routine was to make a stock $0.2 \mathrm{~N}$ acetate buffer at $\mathrm{pH} 5.0$, then make the final solution of $1 \% \mathrm{UA}$ in $0.1 \mathrm{~N}$ buffer, the $\mathrm{pH}$ of which became 5.3. An attempt to readjust the $\mathrm{pH}$ to 5.0 of the UA solution resulted in damaging the appearance of silver particles (small arrows in Fig. 9d). The particles became light grey, and appear to be on the verge of vanishing. Thus, the UA en bloc staining may also contribute to the disappearing of the silver particles. Adjusting the $\mathrm{pH}$ to 6.0 protects the integrity of the silver particles (Fig. 9b), but the contrast of the sample became so low that there is no longer any benefit of this UA en bloc staining step.

Because "greyish" silver particles were still occasionally seen in samples treated with $1 \%$ UA in $0.1 \mathrm{~N}$ acetate buffer at pH5.3, we tried lowering the concentration and treatment time of the UA solution. Samples treated for overnight with $0.25 \%$ UA had a higher contrast and better preservation of the membranes (Fig. 10a) than samples treated for $1 \mathrm{hr}$ (Fig 10b). However, the overnight treatment decreased labeling efficiency. In one experiment, labeling density for SynGAP, a Ras GTPase activating protein highly enriched in the PSD [22], in an overnight-treated sample was $56 \%$ of that of the 1 hr-treated sample (Fig. 10c). We suspect that some of the silver-enhanced particles disappeared during the overnight treatment. Thus, our routine treatment now is to en bloc stain with $0.25 \%$ UA for $1 \mathrm{hr}$ at $4^{\circ} \mathrm{C}$.

\section{Quality of silver particles related to sectioning and counter staining}

Over the years, we noticed that the silver particles in thin sections of some samples appeared smudged as if the silver diffused out of the particles (Fig. 11a). Eventually, we traced this smudging of silver particles to the cleaning of the diamond knife used to cut thin sections. The same block cut with two different diamond knives could result in different looking silver particles. A knife that had been cleaned with ethanol prior to cutting thin sections produced smudged silver particles (Fig. 11a), and this smudging of silver can be remedied by soaking the knife in water over night (Fig. 11b).

Notably, a detergent (any kind of liquid soap)-cleaned diamond knife produced similar looking smudged silver particles, and the smudging effect left by the liquid soap is extremely difficult to remove. Even with careful management on the diamond knives, we sometimes still found smudged silver particles, 
especially in brain tissues. We suspect that the baskets used to hold these tissues during silver enhancement procedures might have been cleaned with detergent, thus, smudged the silver particles.

By reexamining archived grids from past years, it is clear that the quality of the silver-enhanced particles in thin sections all eventually deteriorated with time. A few examples are shown in Fig. 12. The shape of the silver enhanced particle was typically roundish with sharp edges immediately after sectioning (Fig. 12, left column), but deteriorated into irregular shaped, fuzzy particles with time (Fig. 12, right column). The degree of deterioration typically progressed with time, but both the speed and degree of deterioration were variable among different experiments, probably due to the different batches and/or age and handling of silver enhancement kits. Counterstaining of thin sections appears to somewhat protect the silver particles from deteriorating and could prolong the usable life of the thin sectioned grids.

In order to image the silver particles at their best condition, we counterstain thin sections the next day after they were cut, and examine the stained sections as soon as possible, at least within days. However, if the silver particles deteriorated in the sections, the same block can be cut further for more thin sections. The deterioration of the silver particles appears to be related to the exposed cut face of the block because the initial thin sections recut from the face of a previously cut block also contained deteriorated silver particles, yet the deeper sections of the same block yielded intact looking particles.

\section{Considerations on Interpretation of immunogold labeling results}

One of the crucial issues for any immunolabeling study is the specificity of the antibody used to label the protein of interest. Many different validation protocols are summarized in reviews [25, 26], but crossreactive antibodies still pose problems due to lack of multiple approaches in verifications.

For immunogold labeling studies at the EM level, the simplest way to verify the specificity of an antibody is to carry a control sample where the primary antibody is omitted. In these control samples, there should only be background particles scattered randomly. If a primary antibody worked, specific signals should be localized to the expected sites with high precision. Furthermore, immunogold labeling at the EM level has an advantage of being able to use different antibodies to serve as controls for each other. For example, four different antibodies in Fig. 13 were each specifically localized to different sites which may be difficult to differentiate at the LM level, but can be unequivocally distinguished at the EM resolution level. Synaptophysin, an SV membrane protein, was localized to SVs in presynaptic terminals (Fig. 13 a, b) [11], while bassoon, an active zone cytomatrix protein, is localized at the active zone within $100 \mathrm{~nm}$ of the presynaptic membrane (Fig. 13c, d) [10]. On the postsynaptic side, PSD95, a postsynaptic density protein, is localized immediate adjacent to the postsynaptic membrane (Fig. 13 e, f) [3], while shank, a PSD scaffold protein, is located to the deeper layer of the PSD (Fig. $13 \mathrm{~g}, \mathrm{~h}$ ) [24]. Each of these antibodies were specifically localized to their expected sites without cross-labeling other cellular elements.

Another advantage of immunogold labeling at the EM level is the precise localization of transmembrane proteins to their epitopes on either side of the membrane. For example, one antibody made against the $\mathrm{N}$ terminus of the NMDA receptor (NR2B subunit) was localized to the extracellular side of the postsynaptic 
membrane (Fig 14 a) [27], and another antibody made against the C-terminus was localized to the cytosolic side of the membrane (Fig. 14b). The same extracellular NR2B antibody was correctly localized to the lumen of the endoplasmic reticulum (ER, Fig. 14c), so is another antibody against the N-terminus of an AMPA receptor, GluR2 (Fig. 14d) [17]. Two other antibodies made against the cytosolic epitopes of ER membrane proteins, ryanodine receptor and Ip3 receptor, are localized to the cytosolic side of the ER membranes (Fig.14 e, f) [28].

It should be cautioned that some antibodies could cross react to additional, unintended epitopes [25]. For example, one antibody against the homer 2 subfamily of the homer protein, a PSD scaffold protein [23], was correctly localized to the deeper layer of the PSD (Figs. 15a, b). However, this antibody was also localized to other locations unrelated to the expected distribution of homer proteins (Fig. 15c). Signals were seen on the cytosolic side of the nuclear envelope, associated with the nuclear pores (arrows in Fig. 15c). It was determined that this nuclear pore labeling is a cross reaction unrelated to the homer 2 protein because another pan homer antibody which labels all 3 subfamilies of the homer protein did not label the nuclear pore (Fig. 15d). Nevertheless, this Homer 2 antibody is still useful at the EM level because its labeling of the PSD is specific and undisturbed by the additional, non-specific labeling of the nuclear pores, which is distant and distinct from the PSDs.

Another example of an antibody localized to unintended organelles is shown in Fig. 16. An antibody against VGluT (vesicular glutamate transporter), an SV membrane protein specific for glutamate uptake [29], was correctly localized to SVs of glutamatergic synaptic terminals (Fig. 16a) and not to SVs of GABAergic inhibitory terminals (Fig. 16b). However, signals were also detected on cytosolic filaments in soma/dendrites (Fig. 16c). Since VGluT is an integral membrane protein, the label on filaments (inset of Fig. 16c) must be a cross reaction of this antibody and cannot represent VGluT localization. Although the localization on SV membranes is correct (inset of Fig. 16a), we decided not to use this antibody because the cross reaction was too widespread and can potentially obscure the correct signals.

\section{Quantification considerations}

One of the advantages of immunogold labeling is that the labeling density is quantifiable by counting individual particles [5]. However, several issues can affect the validity of quantification. For example, immunolabeling reagents can typically penetrate throughout the monolayer cells of dissociated cultures, but brain tissues present a penetration issue due to tightly packed processes and narrow extracellular space, both of which are not conducive to penetration and diffusion of reagents. To guard against this penetration gradient issue, our routine practice is to cross section immunogold-labeled brain slices and to examine the cut edges for labeling (Fig. 17).

There is a steep downward gradient of label from the cut edge of the tissue toward the center of the brain slices (Fig. 17c-e). Although the depth of labeling and the steepness of the gradient can vary by different perfusion fixations or by different primary antibodies, the highest labeling density was consistently seen in the most superficial 1-2 $\mu \mathrm{m}$ of the brain slices. Thus, for quantification projects using perfusion-fixed 
brains, sampling is restricted to within $1 \mu \mathrm{m}$ of the cut edges. It would not be a valid comparison if sampling were collected from different depth of the slices where labeling efficiencies are vastly different.

Another quantification issue is in the interpretation of aggregated signals. Tightly aggregated proteins can appear as dark material legitimately labeled with multiple signals. For example, CaMKII is shown to self-aggregate into clusters (Fig. 18 a, d) [30], and chromogranin A is known to aggregate in high concentrations in the dense core of dense core vesicles (Fig. 18 b, e) [31]. However, tightly clustered particles could be artifacts, especially when there was no evidence of protein aggregates association with these clusters of particles (Fig. 18 c, f). Such clustering of particles is likely caused by clumping of secondary antibodies and could be miscounted as multiple signals. This possibility can be easily tested by using a known, good secondary antibody in parallel experiments for comparison. The lot of secondary antibody with high degree of clumping should be discarded because it cannot be used for quantification of labeling density.

Interestingly, the protein aggregates in some samples appeared denser than others, and the core of the aggregates lacked labeling (d, e). The CaMKII cluster in (d) was from a sample treated with NMDA for 3 min, where CaMKII molecules might have formed a very tight aggregation that impeded the penetration of labeling reagents. Whereas the CaMKII cluster in (a) was from a sample treated with glycine for 2 min, where the CaMKII molecules could be aggregated with a looser configuration, more conducive to penetration of reagents. On the other hand, the dense core in (e) was from a 6-day old culture, where the dense core vesicles could be more mature and the dense core was more densely packed than the ones in (b), which were from a 3-day old culture. Nevertheless, these examples illustrate that counting labels from $(d, e)$ may not truly reflect the number of epitopes present in these tightly packed protein aggregates.

\section{Conclusions}

In conclusion, the present paper presented means to optimize the fixation and other treatment conditions for each antibody, summarized potential pitfalls and remedies of this technique, and discussed caveats on interpretation of labeling results. If carried out carefully, this pre-embedding immunogold labeling methods is still one of the best tools in localization of specific proteins at the ultrastructural level which can be used to study the redistribution of proteins in neuronal tissues under different stimulation conditions.

\section{Declarations}

\section{Acknowledgements}

We thank Christine A. Winters for supplying dissociated hippocampal cultures of consistent high quality, and Dr. Tom Reese for generous support, both for over 30 years. We are grateful to Drs. Paul Gallant and Milton Brightman for expertly perfusion-fixed brains over the years, and Dr. Ayse Dosemeci for critical 
reading of the manuscript. We thank Drs. Thorkil Ploug and Evelyn Ralston for bringing the first immunogold labeling project into our EM Facility.

\section{Funding}

Supported by National Institute of Neurological Disorders and Stroke (NINDS) intramural funds.

\section{Availability of data and material}

The datasets generated and/or analyzed during the current study are available from the corresponding author on reasonable request.

\section{Authors' contributions}

All authors participated in the design and carried out the experiments, as well as the writing of the manuscript. All authors have read and approved the final manuscript.

\section{Ethics approval}

The animal protocol was approved by the National Institute of Neurological Disorders and Stroke Animal Use and Care Committee (Animal protocol Number: ASP1159) and conforms to NIH guidelines.

\section{Consent for publication}

Not applicable.

\section{Competing interests}

The authors declare that they have no competing interests.

\section{References}

1. Chan J, Aoki C, Pickel VM. Optimization of differential immunogold-silver and peroxidase labeling with maintenance of ultrastructure in brain sections before plastic embedding. J Neurosci Methods. 1990; 33: 113-27.

2. Tanner VA, Ploug T, Tao-Cheng JH. Subcellular localization of SV2 and other secretory vesicle components in PC12 cells by an efficient method of preembedding EM immunocytochemistry for cell cultures. J Histochem Cytochem. 1996; 44: 1481-8.

3. Dosemeci A, Weinberg RJ, Reese TS, Tao-Cheng JH. The postsynaptic density: there Is more than meets the eye. Front Synaptic Neurosci. 2016; 8: 23.

4. Tao-Cheng JH. Activity-dependent redistribution of CaMKII in the postsynaptic compartment of hippocampal neurons. Mol Brain. 2020; 13: 53. 
5. Robinson JM, Takizawa T, Vandré DD, Burry RW. Ultrasmall immunogold particles: important probes for immunocytochemistry. Microsc Res Tech. 1998; 42: 13-23.

6. Hainfeld JF, Powell RD. New frontiers in gold labeling. J Histochem Cytochem. 2000; 48: 471-80.

7. Hacker GW, Grimelius L, Danscher G, Bernatzky G, Muss W, Adam H, Thurner, J. Silver acetate autometallography: an alternative enhancement technique for immunogold-silver staining (IGSS) and silver amplification of gold, silver, mercury and zinc in tissues. J. Histotechnol. 1988; 11: 213221.

8. Burry RW, Vandré DD, Hayes DM. Silver enhancement of gold antibody probes in pre-embedding electron microscopic immunocytochemistry. J Histochem Cytochem. 1992; 40: 1849-56.

9. Lu Z, McLaren RS, Winters CA, Ralston E. Ribosome association contributes to restricting mRNAs to the cell body of hippocampal neurons. Mol Cell Neurosci. 1998; 12: 363-75.

10. Tao-Cheng, J.-H. Ultrastructural localization of active zone and synaptic vesicle proteins in a preassembled multi-vesicle transport aggregate. Neuroscience 2007; 150: 575-584.

11. Tao-Cheng JH. Immunogold labeling of synaptic vesicle proteins in developing hippocampal neurons. Mol Brain. 2020 ;13: 9.

12. Tao-Cheng JH, Gallant PE, Brightman MW, Dosemeci A, Reese TS. Structural changes at synapses after delayed perfusion fixation in different regions of the mouse brain. J Comp Neurol. 2007; 50: 731-40.

13. Dosemeci A, Burch A, Loo H, Toy D, Tao-Cheng JH. IRSp53 accumulates at the postsynaptic density under excitatory conditions. PLoS One. 2017; 12: e0190250.

14. Tao-Cheng JH, Toy D, Winters CA, Reese TS, Dosemeci A. Zinc Stabilizes Shank 3 at the Postsynaptic Density of Hippocampal Synapses. PLoS One. 2016; 11: e0153979.

15. Zheng X, Gallot G. Dynamics of cell membrane permeabilization by saponins using terahertz attenuated total reflection. Biophys J. 2020; 119: 749-755.

16. Ralston E, Ploug T. Pre-embedding staining of single muscle fibers for light and electron microscopy studies of subcellular organization. Scanning Microsc Suppl. 1996; 10: 249-59.

17. Tao-Cheng JH, Crocker VT, Winters CA, Azzam R, Chludzinski J, Reese TS. Trafficking of AMPA receptors at plasma membranes of hippocampal neurons. J Neurosci. 2011; 31: 4834-43.

18. Mandikian D, Bocksteins E, Parajuli LK, Bishop HI, Cerda O, Shigemoto R, Trimmer JS. Cell typespecific spatial and functional coupling between mammalian brain $\mathrm{Kv} 2.1 \mathrm{~K}^{+}$channels and ryanodine receptors. J Comp Neurol. 2014; 522: 3555-74.

19. Hachiya N, Sochocka M, Brzecka A, Shimizu T, Gąsiorowski K, Szczechowiak K, Leszek J. Nuclear envelope and nuclear pore complexes in neurodegenerative diseases-new perspectives for therapeutic interventions. Mol Neurobiol. 2020; https://doi.org/10.1007/s12035-020-02168-x.

20. Hovius R, Lambrechts H, Nicolay K, de Kruijff B. Improved methods to isolate and subfractionate rat liver mitochondria. Lipid composition of the inner and outer membrane. Biochim Biophys Acta. 1990; 1021: 217-26. 
21. Salio C, Lossi L, Merighi A. Combined light and electron microscopic visualization of neuropeptides and their receptors in central neurons. Methods Mol Biol. 2011; 789: 57-71.

22. Yang Y, Tao-Cheng JH, Reese TS, Dosemeci A. SynGAP moves out of the core of the postsynaptic density upon depolarization. Neuroscience. 2011; 192:132-9.

23. Tao-Cheng JH, Thein S, Yang Y, Reese TS, Gallant PE. Homer is concentrated at the postsynaptic density and does not redistribute after acute synaptic stimulation. Neuroscience. 2014; 266: 80-90.

24. Voskuil JLA, Bandrowski A, Begley CG, Bradbury ARM, Chalmers AD, Gomes AV, Hardcastle T, LundJohansen F, Plückthun A, Roncador G, Solache A, Taussig MJ, Trimmer JS, Williams C, Goodman SL. The Antibody Society's antibody validation webinar series. MAbs. 2020; 12: 1794421.

25. Uhlen M, Bandrowski A, Carr S, Edwards A, Ellenberg J, Lundberg E, Rimm DL, Rodriguez H, Hiltke T, Snyder M, Yamamoto T. A proposal for validation of antibodies. Nat Methods. 2016; 13: 823-7.

26. Tao-Cheng JH, Yang Y, Reese TS, Dosemeci A. Differential distribution of Shank and GKAP at the postsynaptic density. PLoS One. 2015; 10:e0118750.

27. Tao-Cheng JH, Azzam R, Crocker V, Winters CA, Reese T. Depolarization of hippocampal neurons induces formation of nonsynaptic NMDA receptor islands resembling nascent postsynaptic densities. eNeuro. 2015; 2:ENEURO.0066-15.2015.

28. Mignery GA, Südhof TC, Takei K, De Camilli P. Putative receptor for inositol 1,4,5-trisphosphate similar to ryanodine receptor. Nature. 1989; 342: 192-5.

29. Fujiyama F, Furuta T, Kaneko T. Immunocytochemical localization of candidates for vesicular glutamate transporters in the rat cerebral cortex. J Comp Neurol. 2001; 435: 379-87.

30. Dosemeci A, Reese TS, Petersen J, Tao-Cheng JH. A novel particulate form of $\mathrm{Ca}(2+) /$ calmodulindependent protein kinase II in neurons. J Neurosci. 2000; 20: 3076-84.

31. Kim T, Tao-Cheng JH, Eiden LE, Loh YP. Chromogranin A, an "on/off" switch controlling dense-core secretory granule biogenesis. Cell. 2001; 106: 499-509.

\section{Figures}



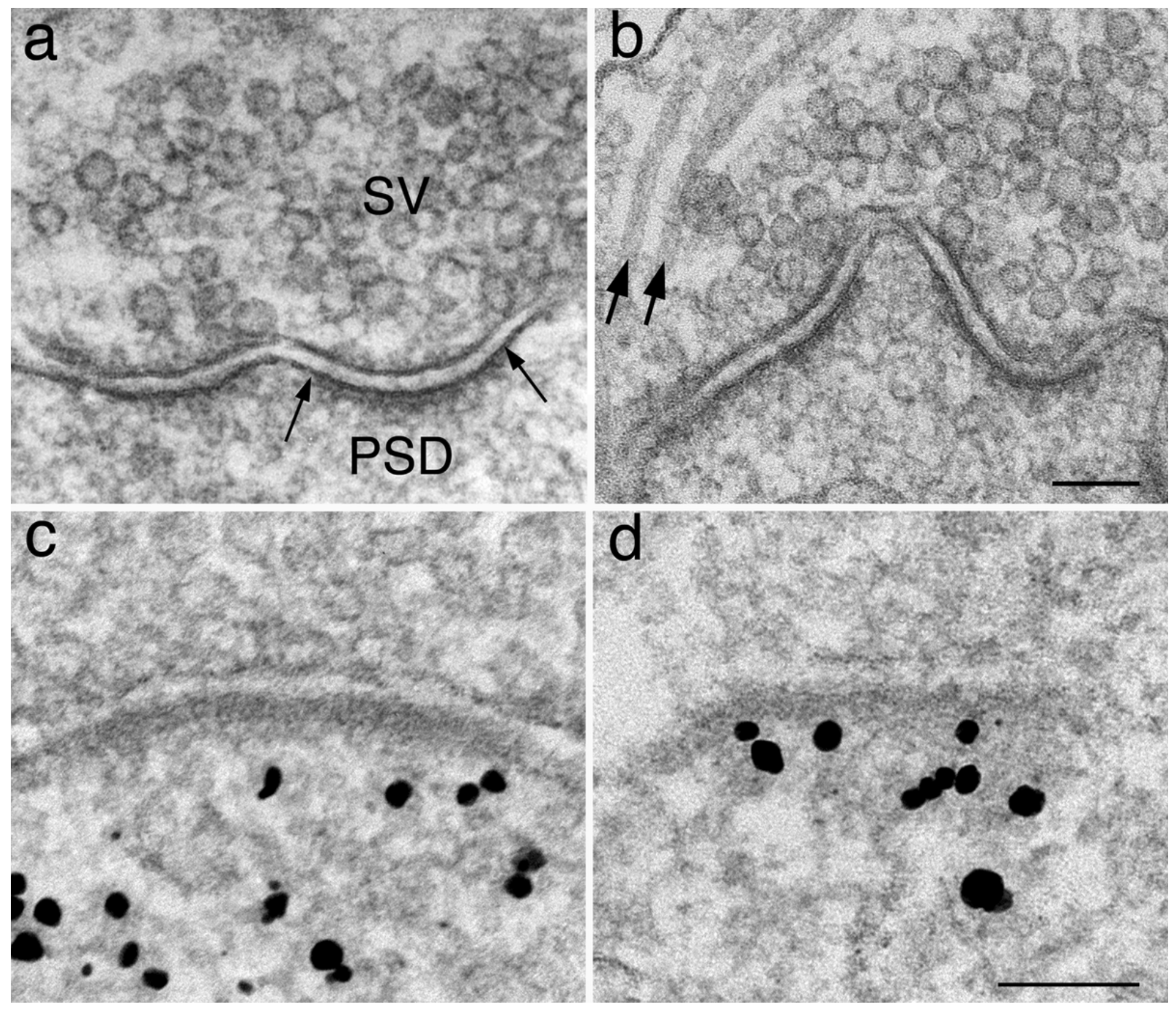

\section{Figure 1}

Comparison on structural preservation upon different fixation conditions and subsequent treatments. Images show synaptic profiles from 3 wk-old dissociated hippocampal cultures without $(a, b)$ or with (c, d) immunogold labeling. Clusters of synaptic vesicles (SV in a) and the postsynaptic density (PSD, edges of which marked with arrows in a) are characteristics of glutamatergic asymmetric synapses. The fixation treatments were 4\% PF in PBS for 45 min (a), 4\% glutaraldehyde in $0.1 \mathrm{M}$ cacodylate buffer at pH 7.4 for 30 min (b), 4\% PF in PBS for 30 min and labeled for CaMKII (c), a cytosolic protein in neurons [4], and $2 \%$ PF in PBS for 15 min and labeled for IRSp53 (d), an actin-associated protein enriched at the PSD [13]. All fixations were carried out at room temperature. Membranes were poorly preserved in (d) due to the lower concentration of PF and the shorter fixation time. Scale bars $=100 \mathrm{~nm}$. 

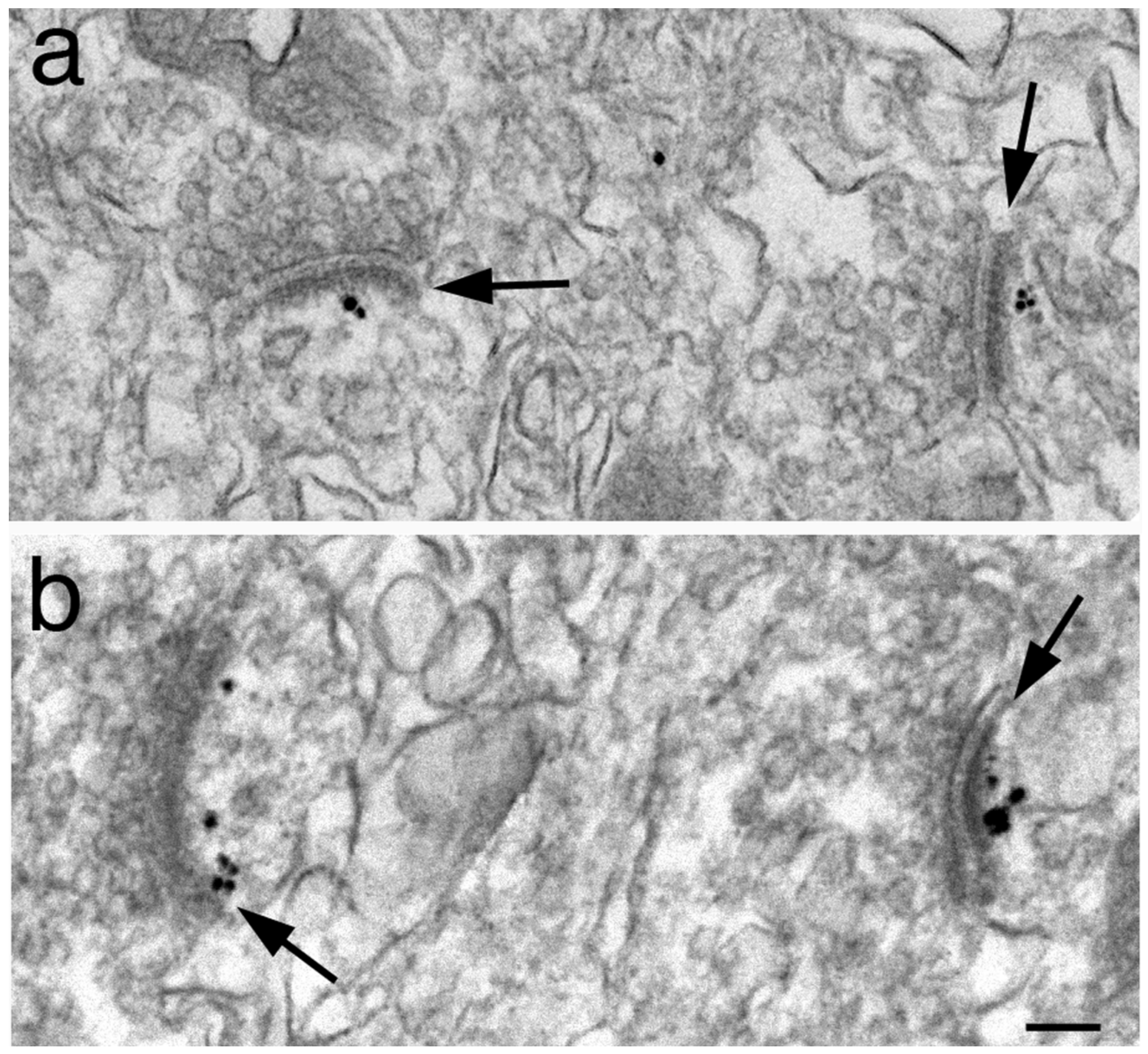

\section{Figure 2}

Perfusion-fixed mouse brains labeled for Shank 3, a PSD scaffold protein [14]. Labeling density at PSDs (arrows) was lower in the brain that was fixed with 4\% PF for 60 min (a) than the one that was fixed for $40 \mathrm{~min}(\mathrm{~b})$. Scale bar $=100 \mathrm{~nm}$. 


\section{saponin}

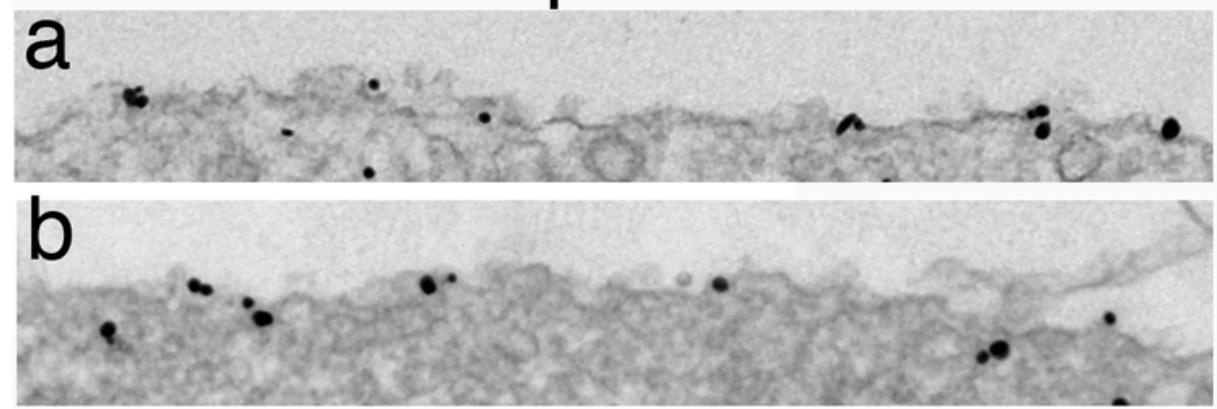

ethanol

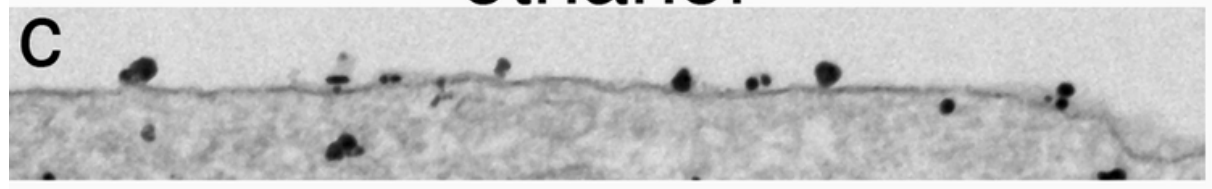

d

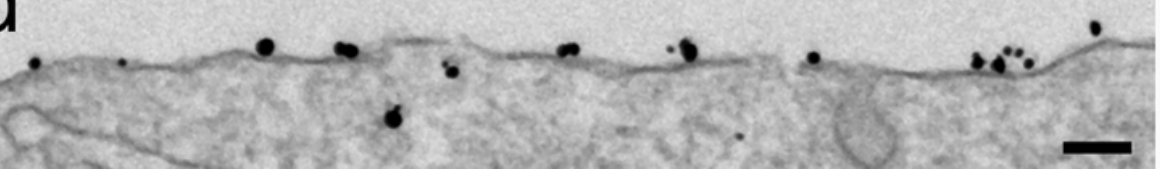

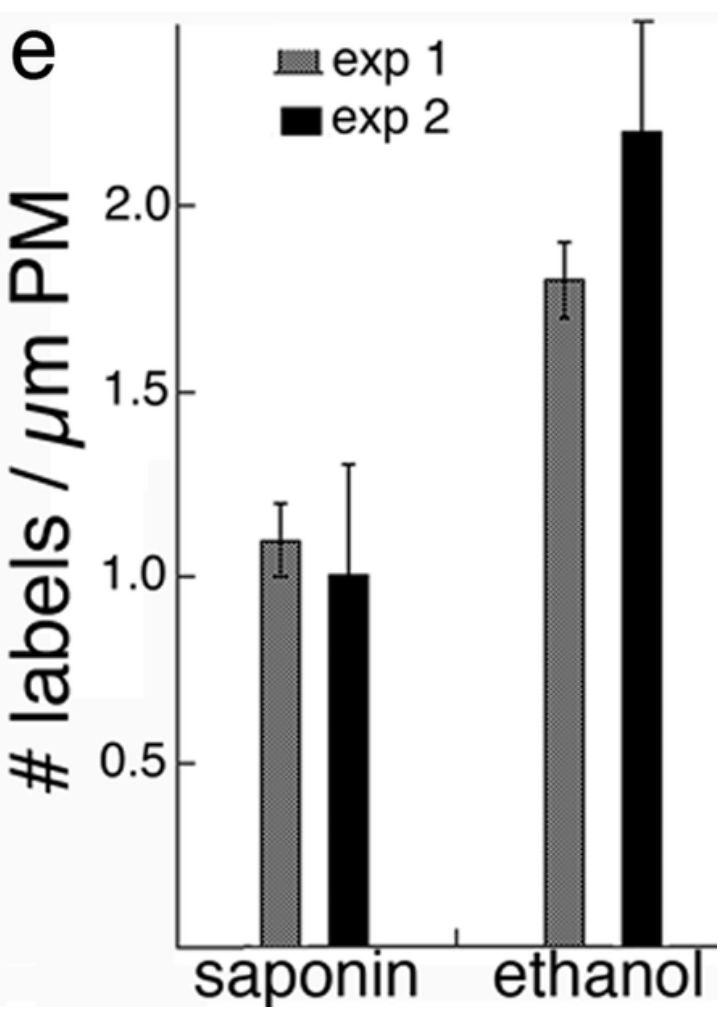

Figure 3

Plasma membranes (PM) of dissociated hippocampal neuronal somas were labeled for GluR2, an AMPA type glutamate receptor of the subtype 2 . Samples were identically fixed but permeabilized differently with saponin $(a, b)$ or ethanol $(c, d)$. Scale bar $=100 \mathrm{~nm}$. (e). Labeling density was different under the two conditions. Data from Additional File 1.
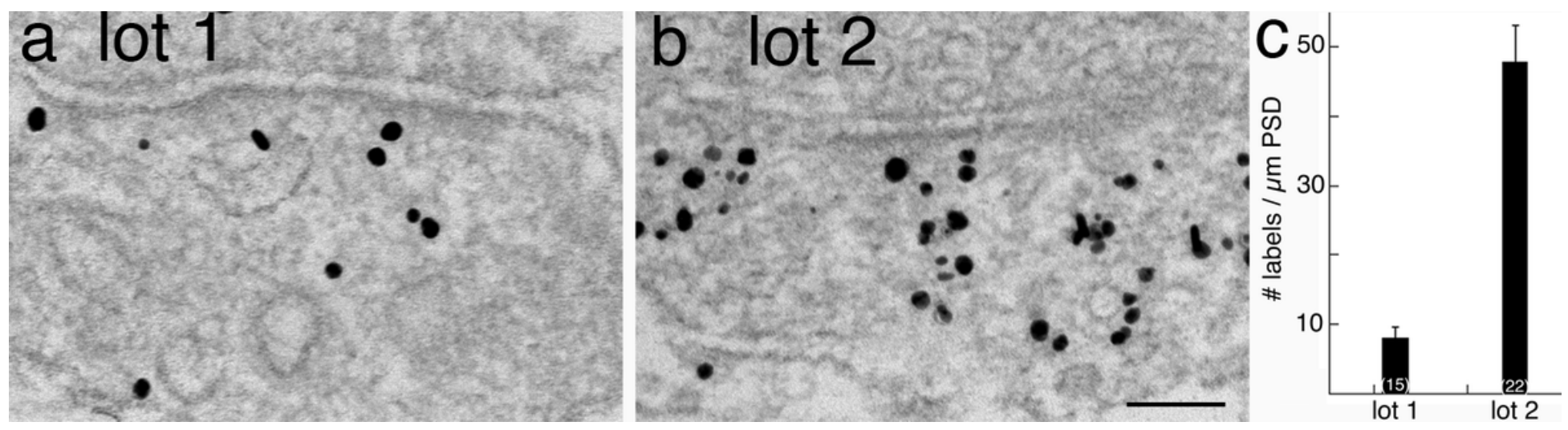

Figure 4

Quality of the secondary antibody affects the labeling efficiency. Dissociated hippocampal cultures were labeled for CaMKII, a cytosolic protein concentrated at the postsynaptic dendritic elements [4]. Labeling density was measured within $120 \mathrm{~nm}$ of the postsynaptic membrane, and was much lower in samples incubated with one lot of secondary antibody (a) than another (b). Scale bar $=100 \mathrm{~nm}$. (c). Labeling densities were significantly different $(P<0.0001$, Student's $t$-test) between the two lots of secondary antibodies. ( $\mathrm{n}$ ) at the bottom of bars represent number of synaptic profiles sampled. 
Label for SV2 in a parallel experiment

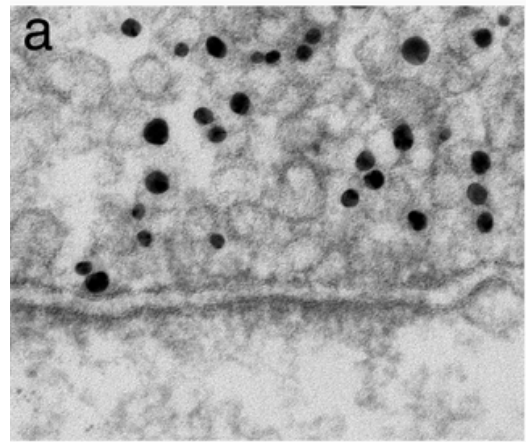

Aurion silver enhancement, $60 \mathrm{~min}$

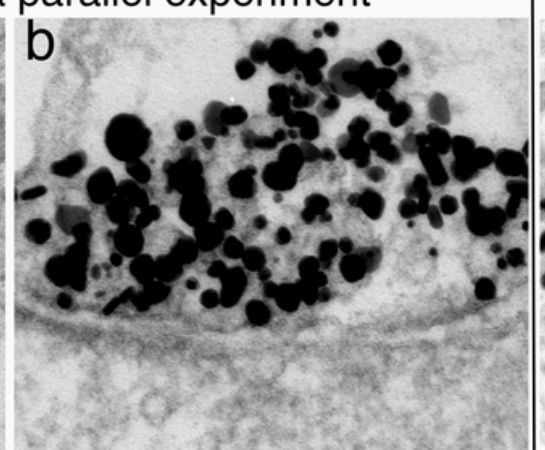

$\mathrm{HQ}$ silver enhancement, 6 min

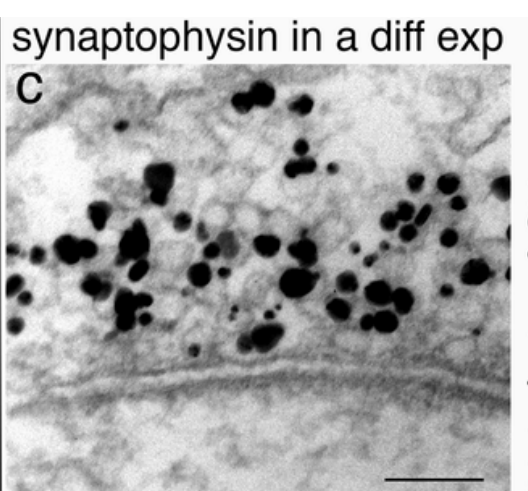

$\mathrm{HQ}$ silver enhancement, $5 \mathrm{~min}$

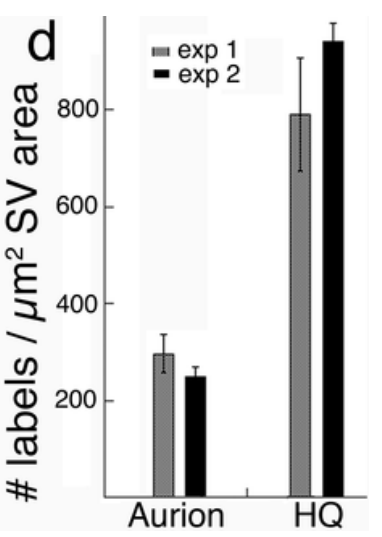

Figure 5

Samples in a and b were labeled for SV2 and treated identically except for the silver enhancement step. Samples treated with the Aurion silver enhancement kit for 60 min showed signals of more or less uniform-sized particles (a), while samples treated with the Nanoprobes HQ silver enhancement kit for 6 min showed signals of heterogeneous sizes at a much higher density (b). Sample in c was from a separate experiment where sample was labeled with synaptophysin, another SV membrane protein, and the time of $\mathrm{HQ}$ silver enhancement was shortened to $5 \mathrm{~min}$, resulting in smaller sized particles. Scale bar $=100 \mathrm{~nm}$. (d) Labeling density of SV2 after Aurion or HQ silver enhancement kit. Data from Additional File 3.
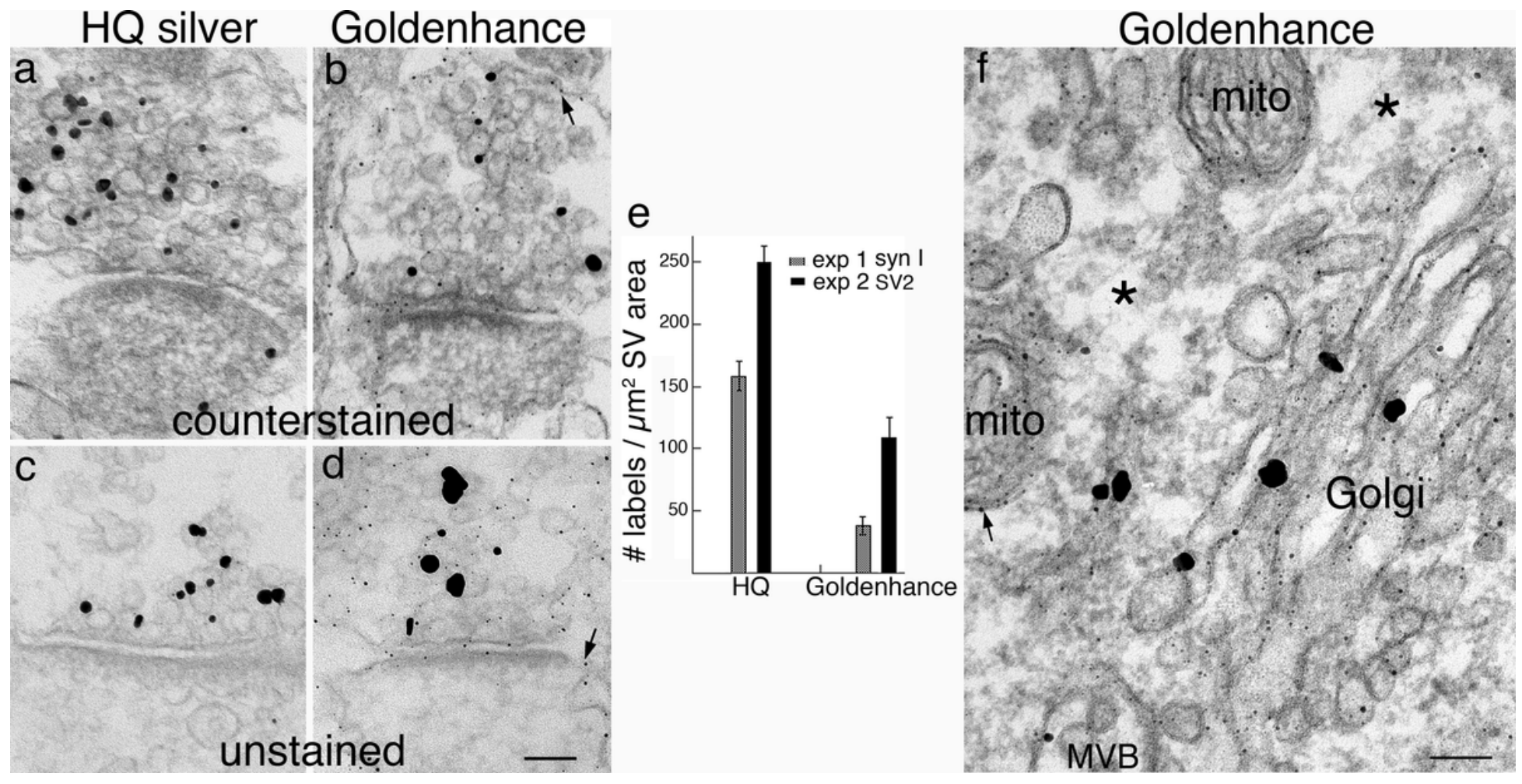

\section{Figure 6}

(a-d) Synapses from dissociated hippocampal neurons labeled with synapsin I, an SV-associated protein [11]. Thin sections were counterstained with UA and lead citrate in a \& b, and unstained in c \& $d$. Labeling 
density on synaptic vesicle clusters is higher after HQ silver enhancement $(a, c)$ than after Goldenhance (b, d). (e) Bar graphs of labeling density from Additional File 4. Notably, in Goldenhance-treated samples $(b, d)$, there were fine particles (small arrows) all over membranes in both stained (b) and unstained (d) sections, indicating that these fine particles are not artifacts of counterstaining. ( $f$ ) A neuronal soma of perfusion-fixed brain labeled with synaptophysin, known to be present at the Golgi complex [11]. This sample was treated with Goldenhance and only the large particles localized on Golgi (large arrows) are real signals. The fine particles present on membranes of Golgi complex, multivesicular body (MVB), and the inner and outer membranes of mitochondria (small arrow in $\mathrm{f}$ ) are artifacts of the Goldenhance reagents. Asterisks $\left.{ }^{*}\right)$ marks the cytosolic area devoid of the fine particles that nonspecifically decorated all membranes. Scale bars $=100 \mathrm{~nm}$. a-d share the same scale bar.

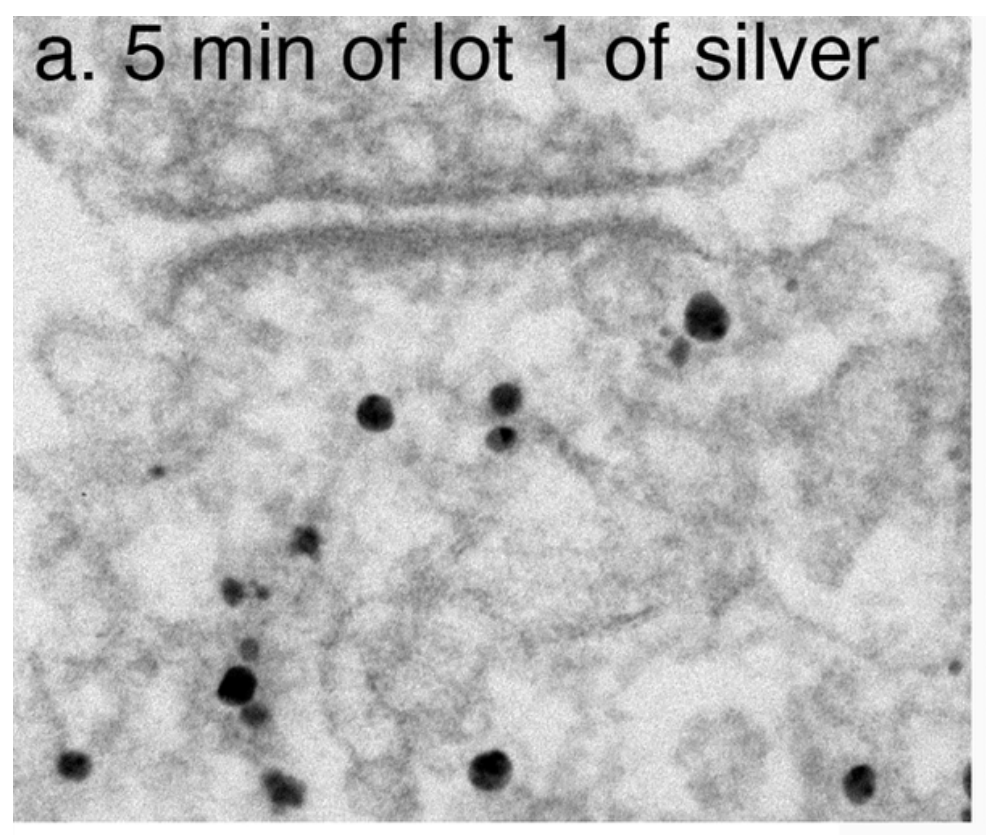

b. 8 min of lot 1 of silver
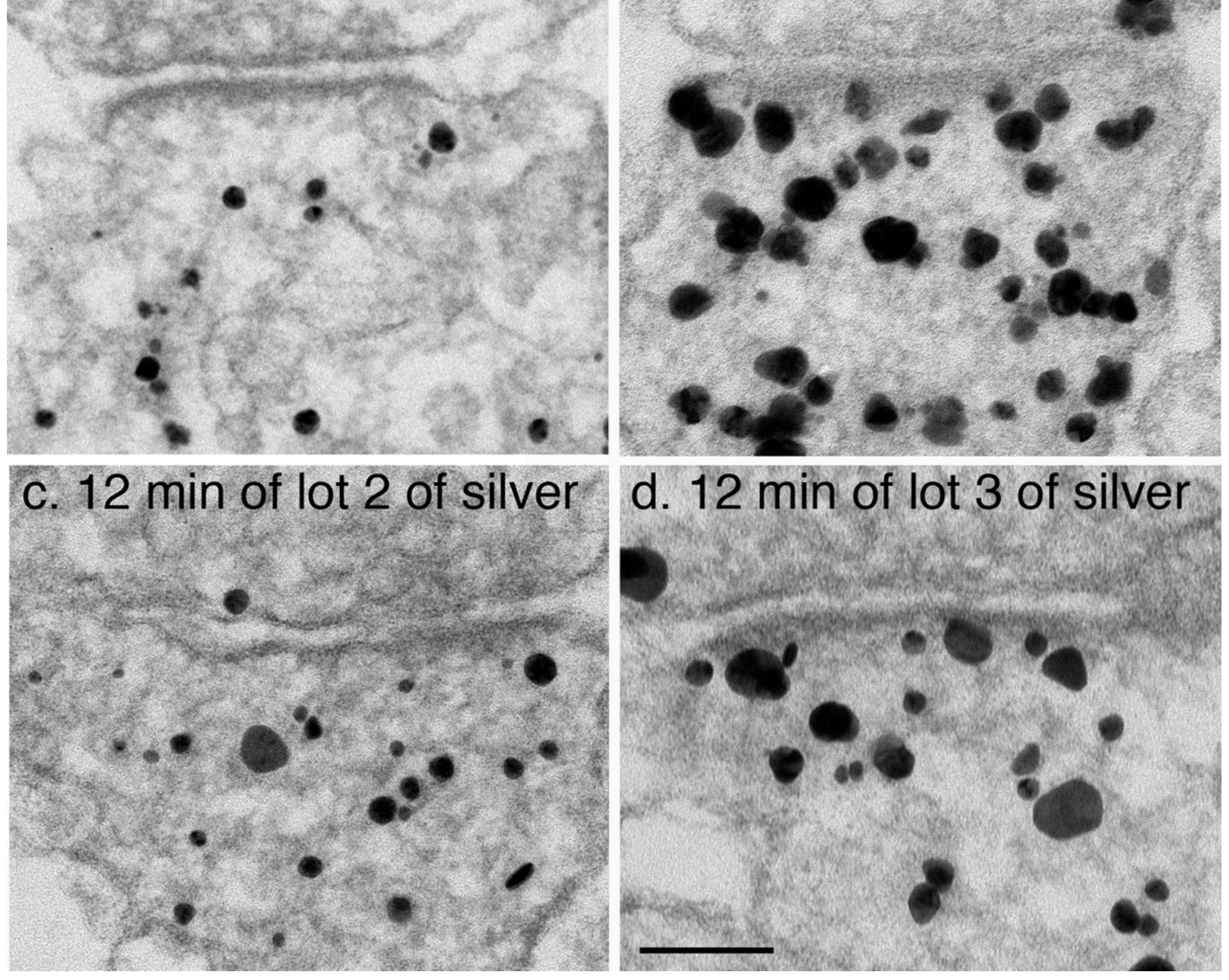

Figure 7 
Synapses in dissociated hippocampal cultures labeled with CaMKII and silver enhanced with different lots of the Nanogold HQ kit. As expected, the size of particles increased with development time $(a, b)$. In (c, d), two different lots of $\mathrm{HQ}$ yielded particles of different size ranges after the same amount of development time. Scale bar $=100 \mathrm{~nm}$.

\section{$1 \%$ osmium}

a
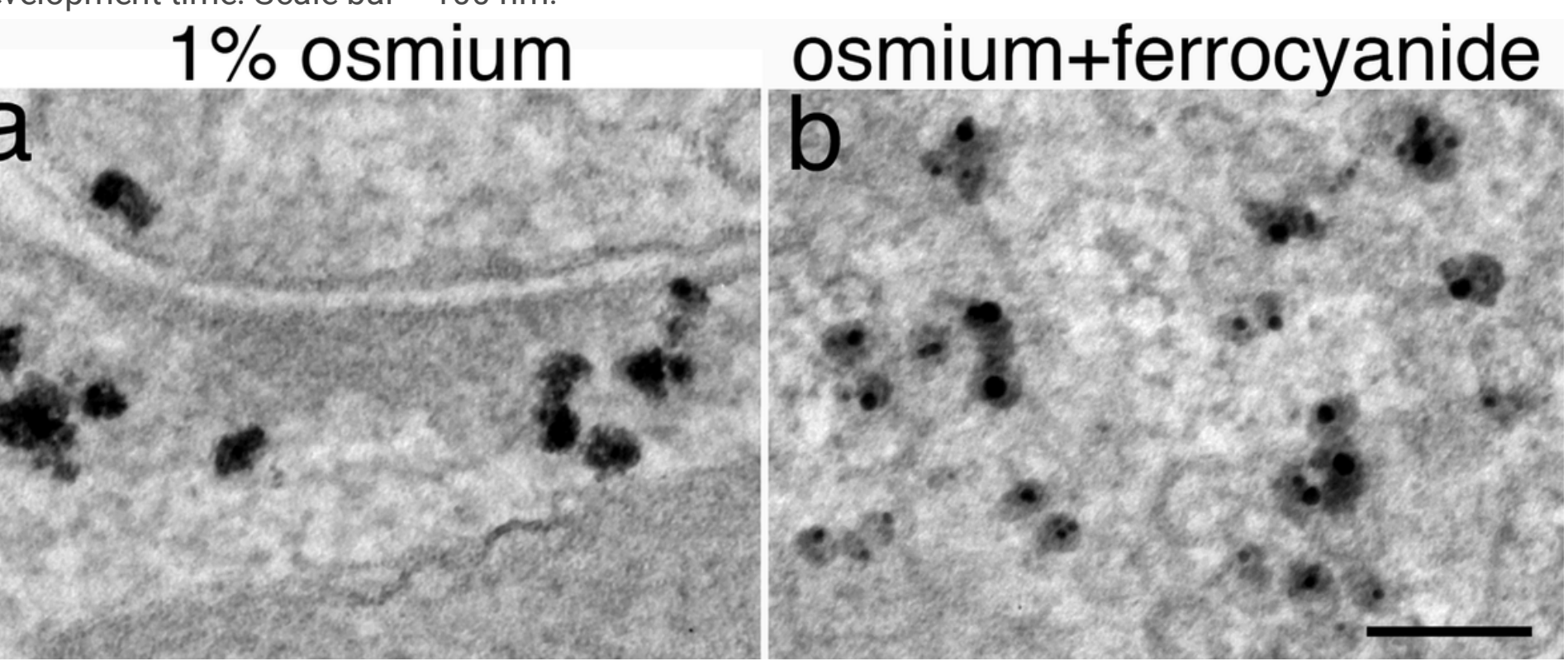

Figure 8

Examples of poor looking silver particles in samples treated with $1 \%$ osmium tetroxide (a) or $1 \%$ osmium $+1 \%$ potassium ferrocyanide (b). Scale bar $=100 \mathrm{~nm}$. 


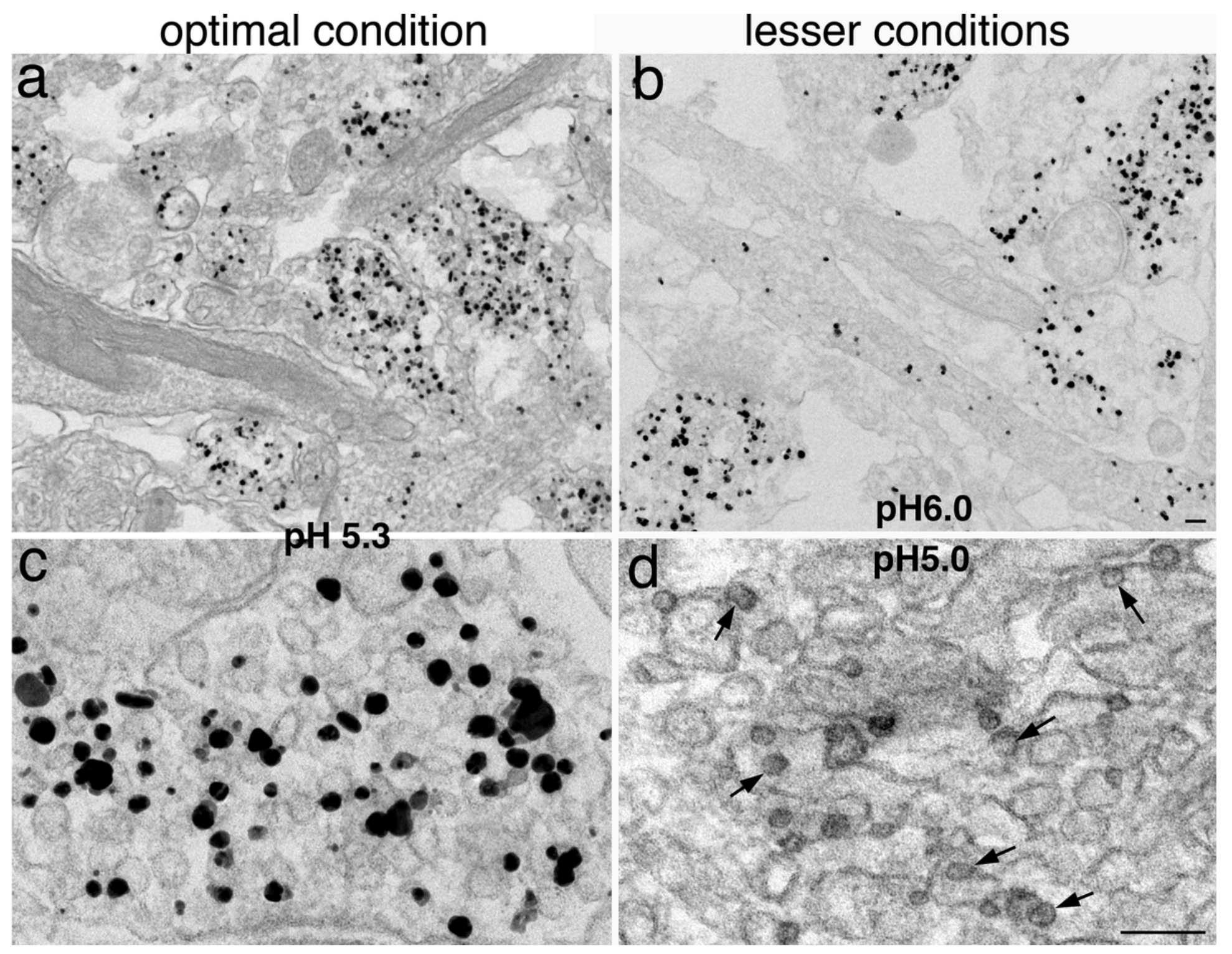

Figure 9

Dissociated hippocampal cultures labeled with SV2. Images have more contrast when samples were en bloc stained with $\mathrm{UA}$ at pH 5.3 (a) than at pH 6.0 (b). Contrast of the image is even stronger when the sample was treated with UA at pH 5.0 (d), but the silver particles became grey shadows (small arrows in d) when compared to the black particles in samples treated with UA at pH 5.3 (c). Scale bars $=100 \mathrm{~nm}$. a \& $b$ shared the same bar, $c$ \& $d$ shared the same bar. 


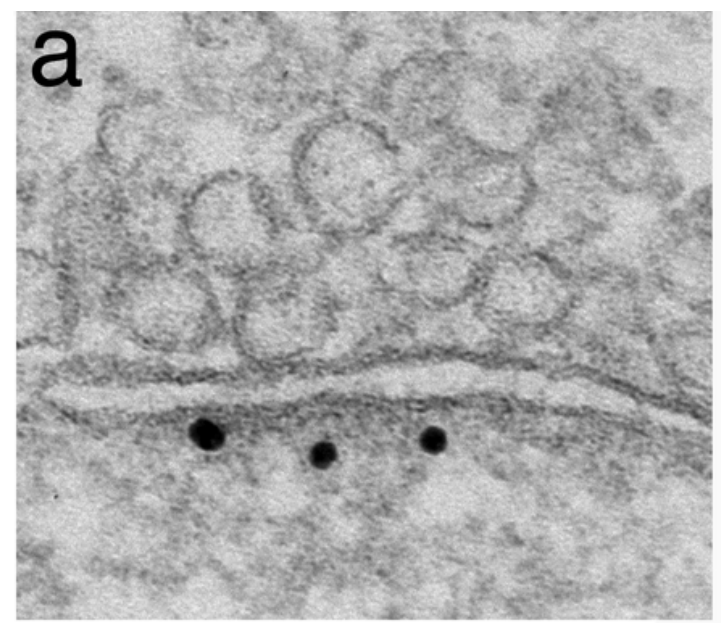

$0.25 \%$ UA overnight

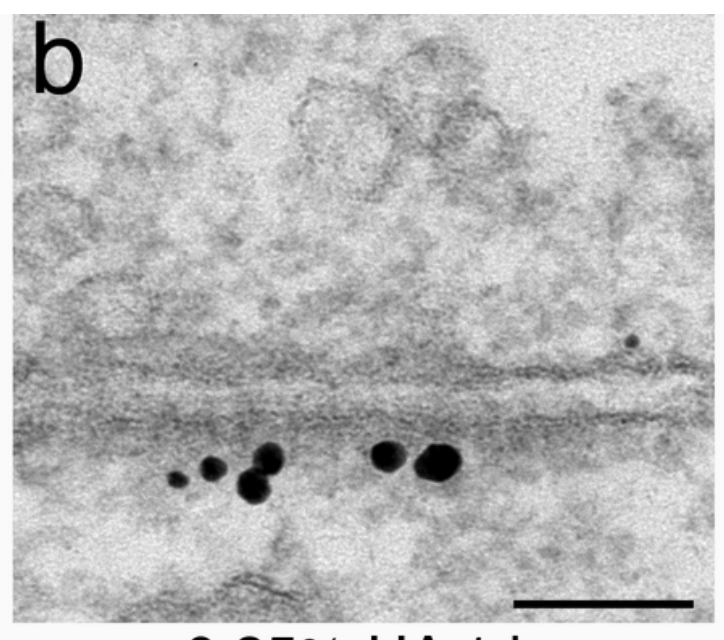

$0.25 \%$ UA $1 \mathrm{hr}$

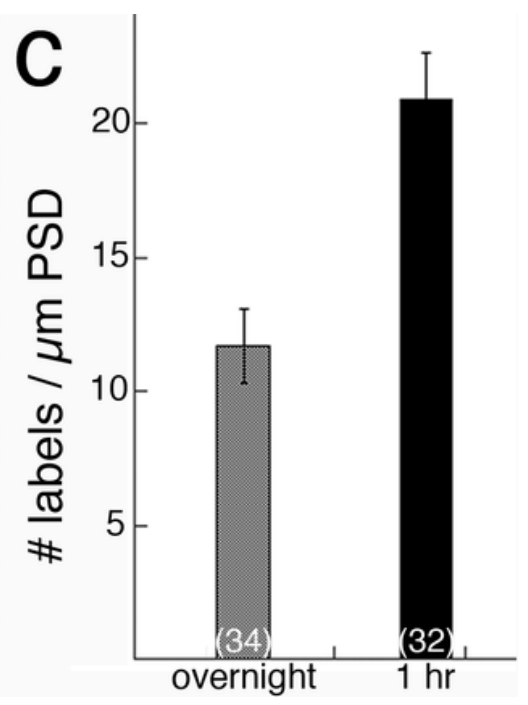

Figure 10

Dissociated hippocampal cultures labeled with SynGAP and treated with $0.25 \%$ UA en bloc for overnight (a) or $1 \mathrm{hr}$ (b). The overnight-treated sample had a higher contrast but lower labeling density at the PSD (c). Scale bar $=100 \mathrm{~nm}$. (c). Number of synaptic profiles measured are listed at the bottom of the bar graphs $(\mathrm{P}<0.005$ by Student's t-test).

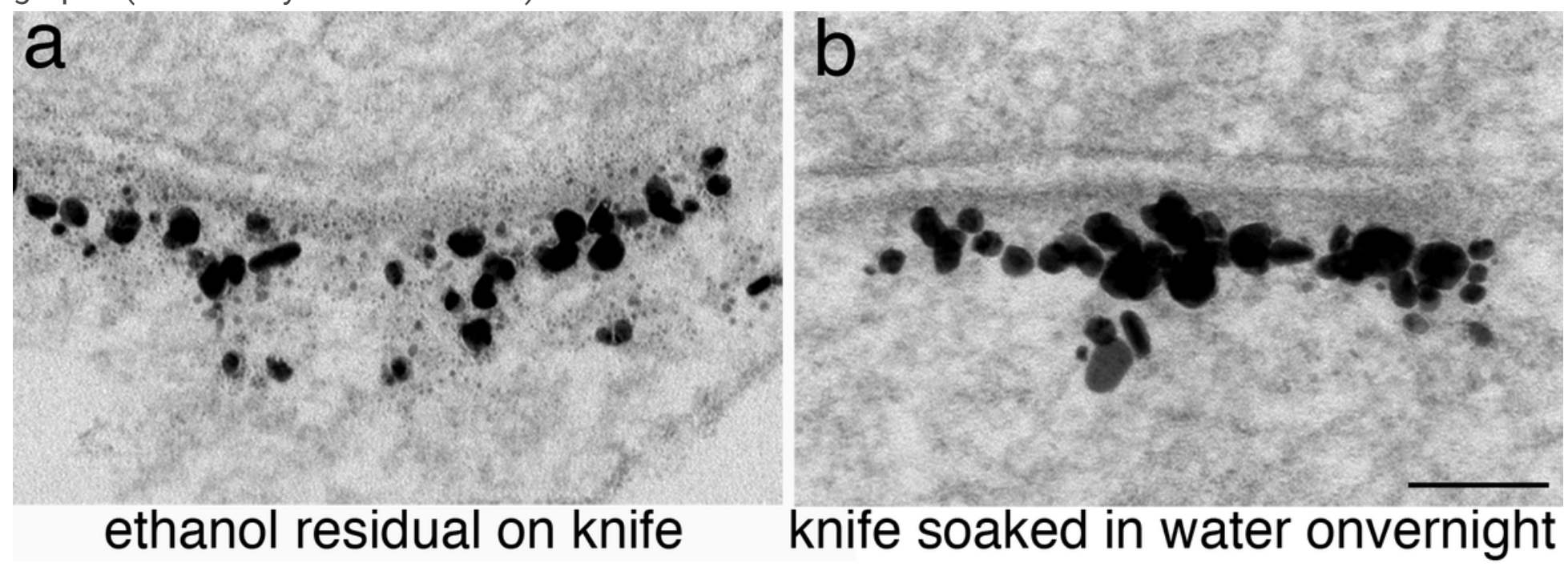

Figure 11

Dissociated hippocampal cultures labeled for Homer $1 \mathrm{~b} / \mathrm{c}$, a scaffold protein at the PSD [23]. When the section was cut with a diamond knife cleaned with ethanol prior to sectioning, the silver particles had a smudged appearance, with numerous fine dots in the vicinity of the particles (a). When the diamond knife cleaned with ethanol was rinsed and soaked in water for at least overnight, the silver particles appeared intact (b). Scale bar $=100 \mathrm{~nm}$. 
immediately after sectioning
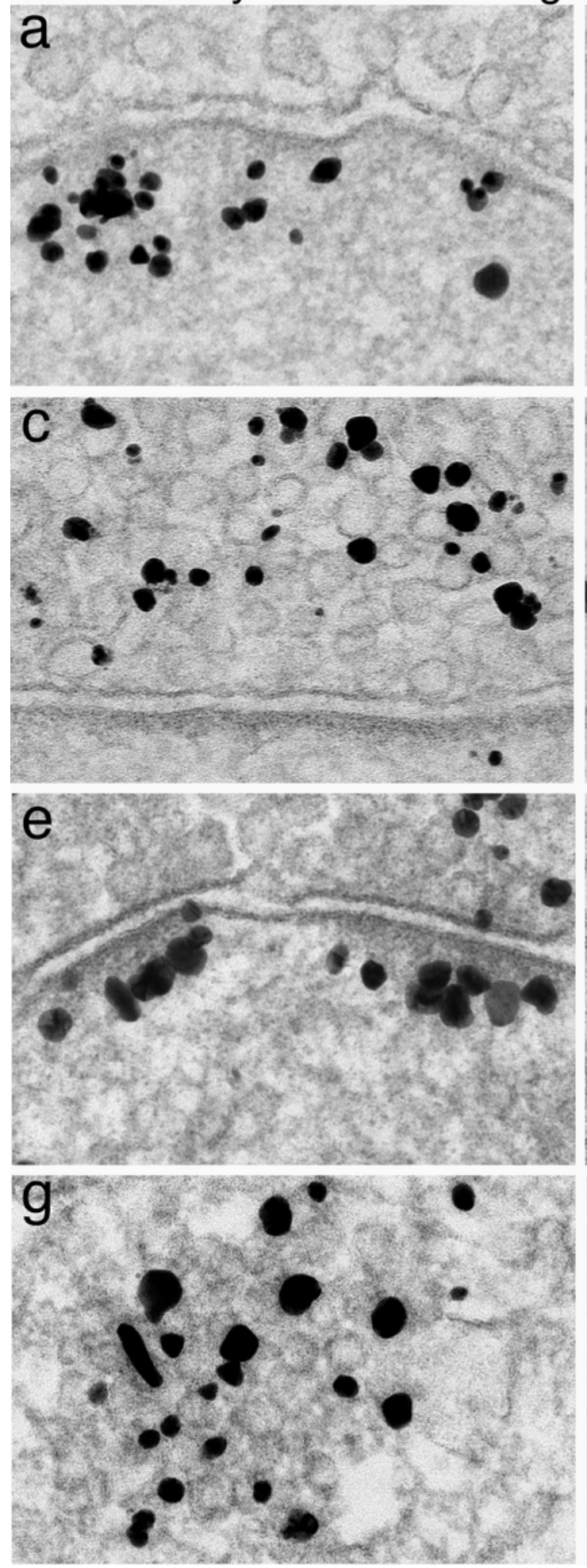

\section{sometime later}

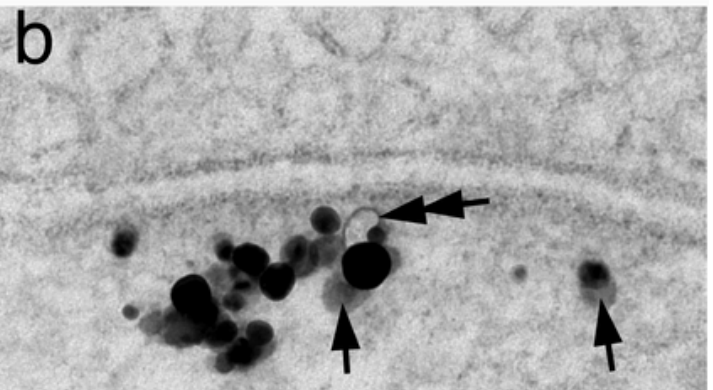

\section{5 wks}
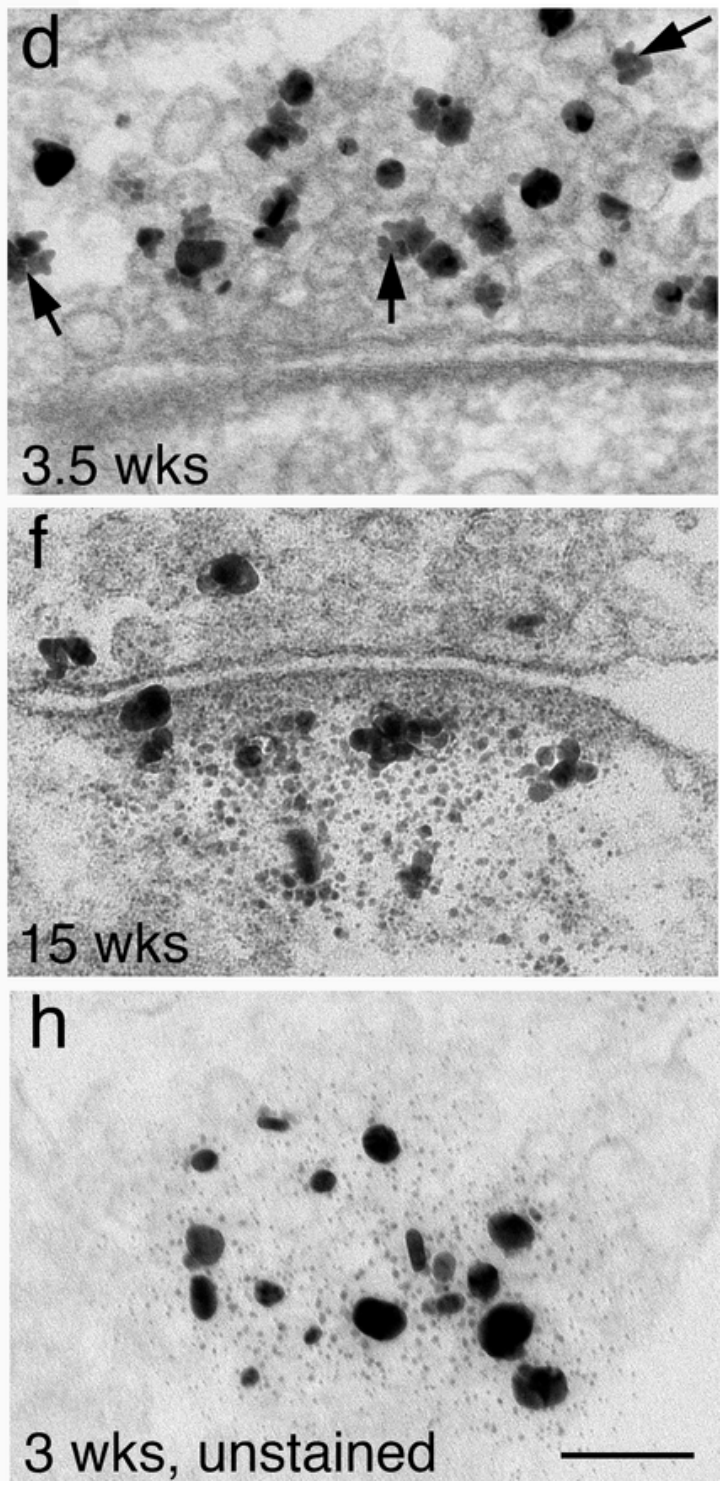

Figure 12

Quality of silver enhanced particles in thin sections deteriorates over time. Sections from the same block of dissociated hippocampal culture examined immediately (left column) and later at different time points (right column): (a, b)-Sample was labeled with Shank 2, a PSD scaffold protein [24]. 5 wks later, some of the particles hollowed (double arrow in b) and some became grey (arrows in b). (c, d)-Sample was labeled with synapsin I, an SV-associated protein [11]. 3.5 wks later, some of the silver particles had 
irregular edges (arrows in d). (e, f)- Sample was labeled with CaMKII [4]. 15 wks later, silver particles became badly smudged (f). (g, h)-Sample was labeled with synapsin I. 3 wks later, the smudge of the silver particles was clearly visible in unstained sections $(h)$, indicating that the smudging of the silver particles is not related to counterstaining. Scale bar $=100 \mathrm{~nm}$.
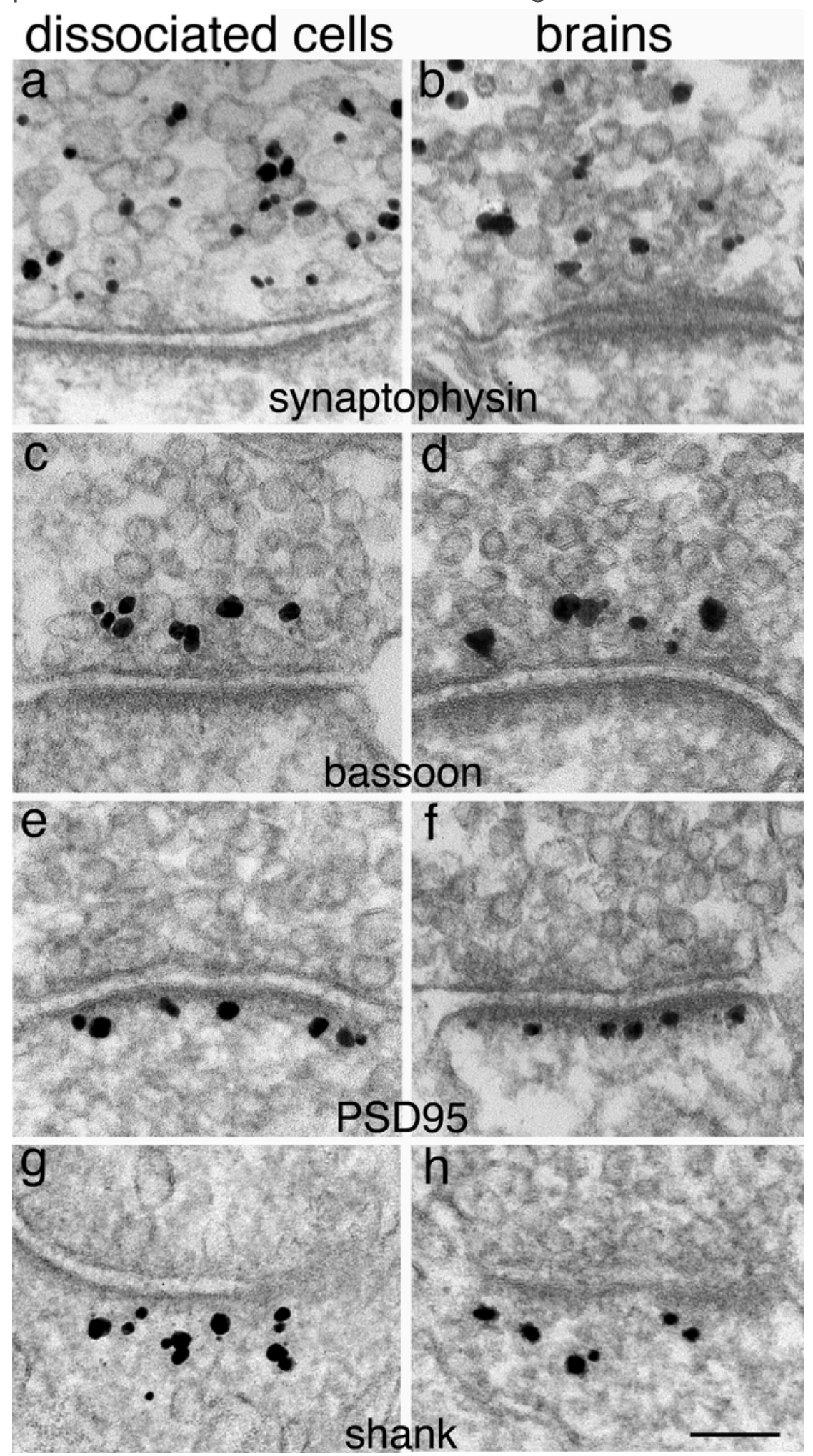

Figure 13 
Dissociated hippocampal culture (left column) and perfusion-fixed mouse or rat brains (right column) labeled with different antibodies. Synaptophsin is localized to synaptic vesicles $(a, b)$, bassoon is localized to active zone cytomatrix (c, d), PSD95 is localized within $30 \mathrm{~nm}$ of the postsynaptic membrane $(e, f)$, and shank is localized within 40-120 nm of the postsynaptic membrane $(\mathrm{g}, \mathrm{h})$. Scale bar $=100 \mathrm{~nm}$.

\section{external epitopes}

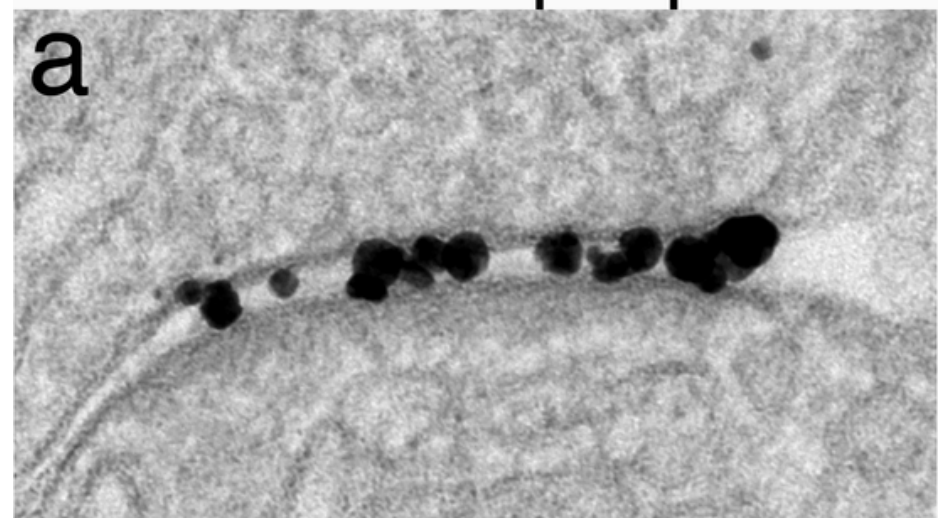

two dofferemt NMDA

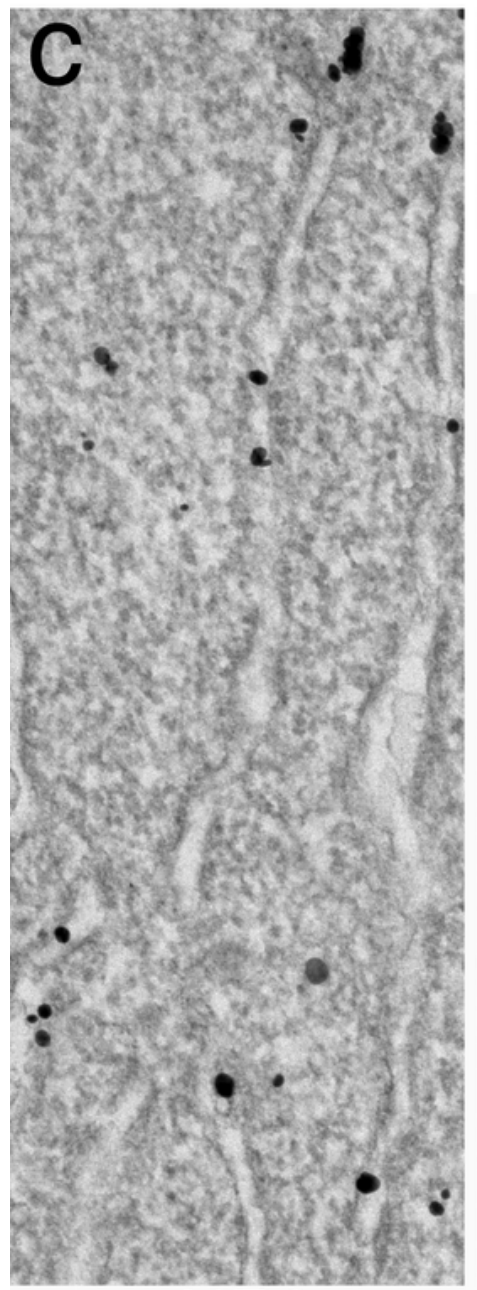

NR2

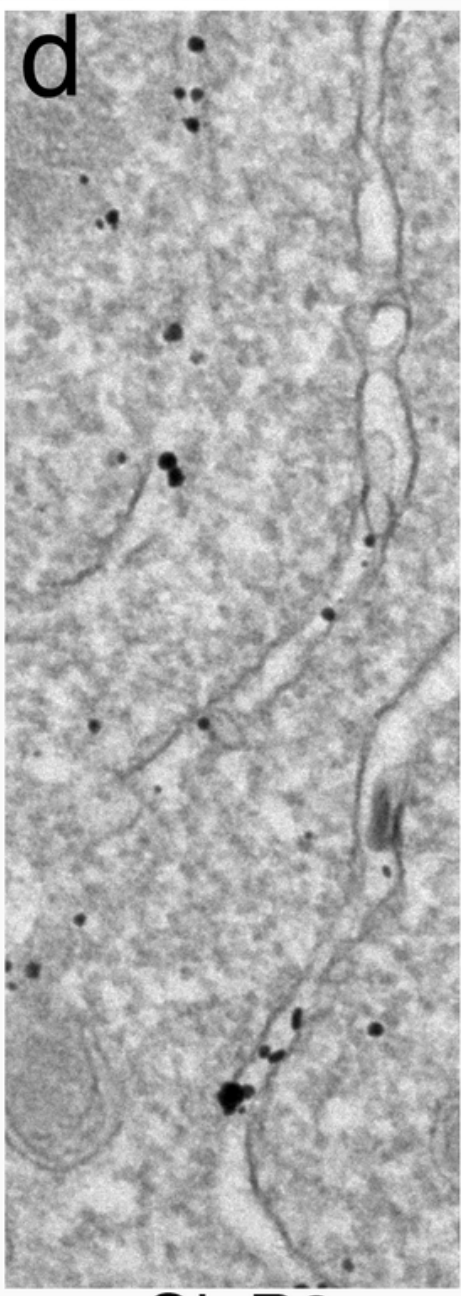

GluR2

\section{cytosolic epitopes}

b

\section{receptor antibodies}

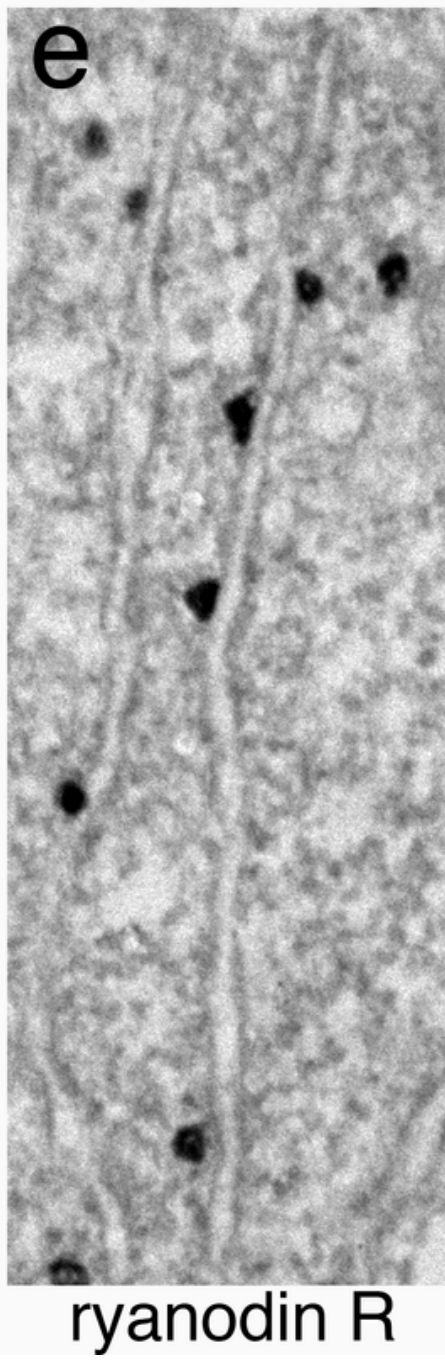

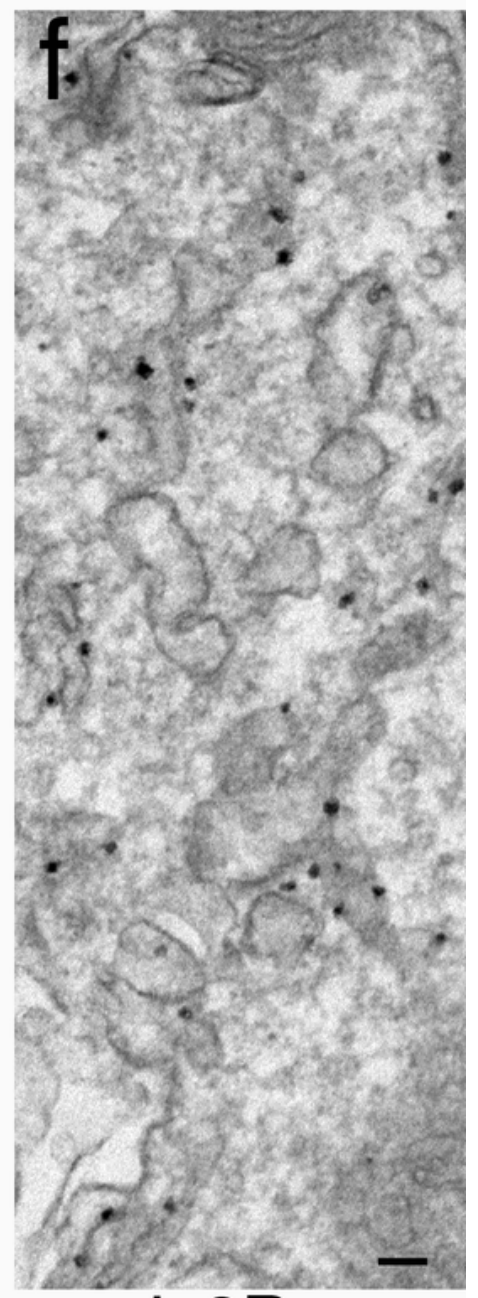

Ip3R

Figure 14 
Verification of specificity of antibodies made against different epitopes of transmembrane proteins. Antibodies made against extracellular (external) epitopes should be localized to the extracellular side of the postsynaptic membrane (a) and the lumen of the ER (c, d), while antibodies made against cytosolic epitopes should be localized to the cytosolic side of the postsynaptic membrane (b) and the cytosolic side of the ER $(e, f)$. Scale bars $=100 \mathrm{~nm}$. a \& b share the same bar, c-f share the same bar. (a-e) from dissociated hippocampal cultures, and (f) from Purkinje soma of perfusion-fixed cerebellum.
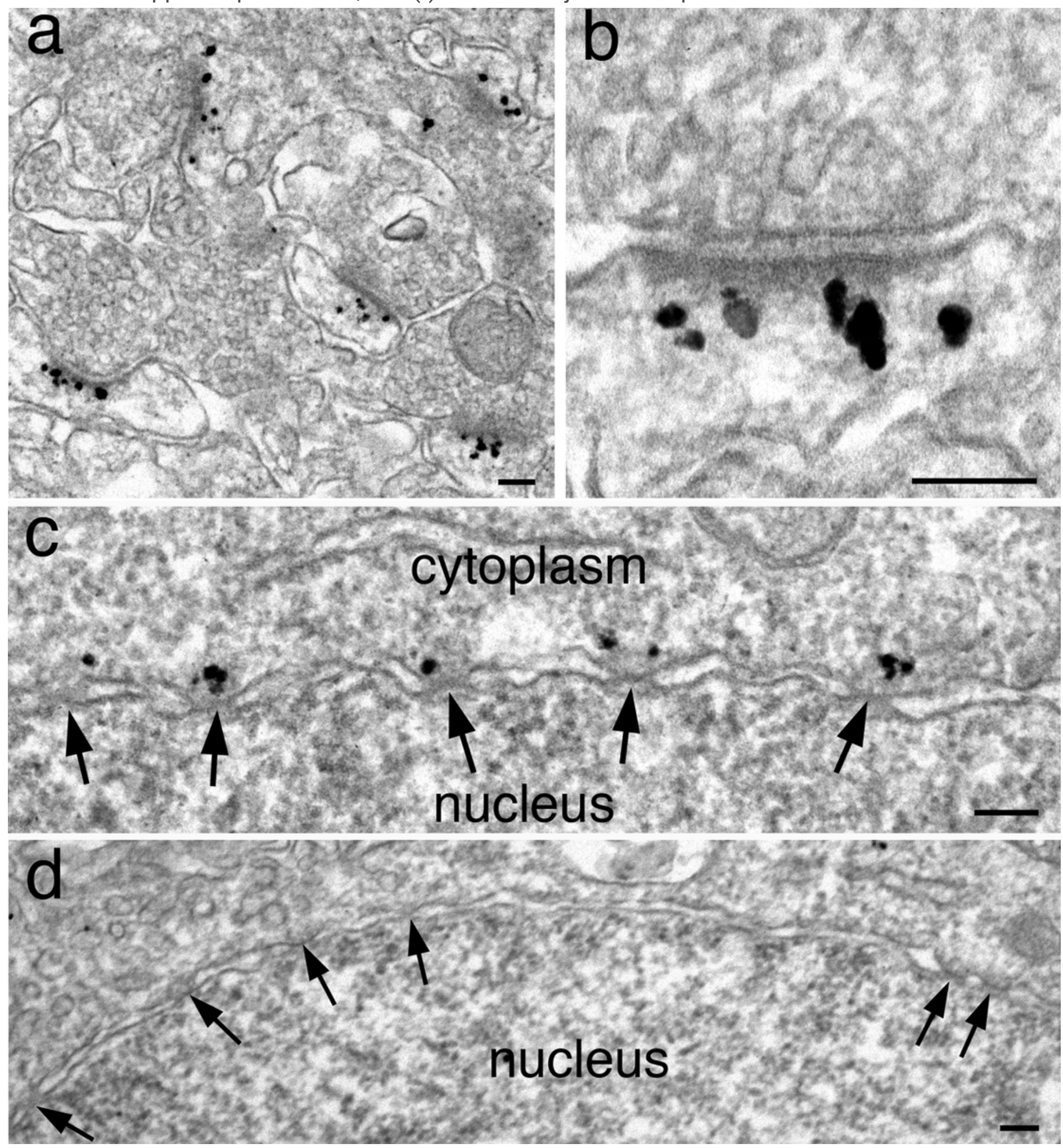

Figure 15 
Perfusion-fixed mouse brains labeled with Homer 2 (a-c) and Homer 1/2/3 (d). Label for a Homer 2 antibody was specifically localized at the PSDs with very little background elsewhere (a), and correctly situated at the deeper layer of the PSD (b). Additional signals of this antibody were also seen associated with the cytoplasmic side of the nuclear pores (arrows in c). However, another pan homer antibody was not detected at the nuclear pore (d). Scale bars $=100 \mathrm{~nm}$.

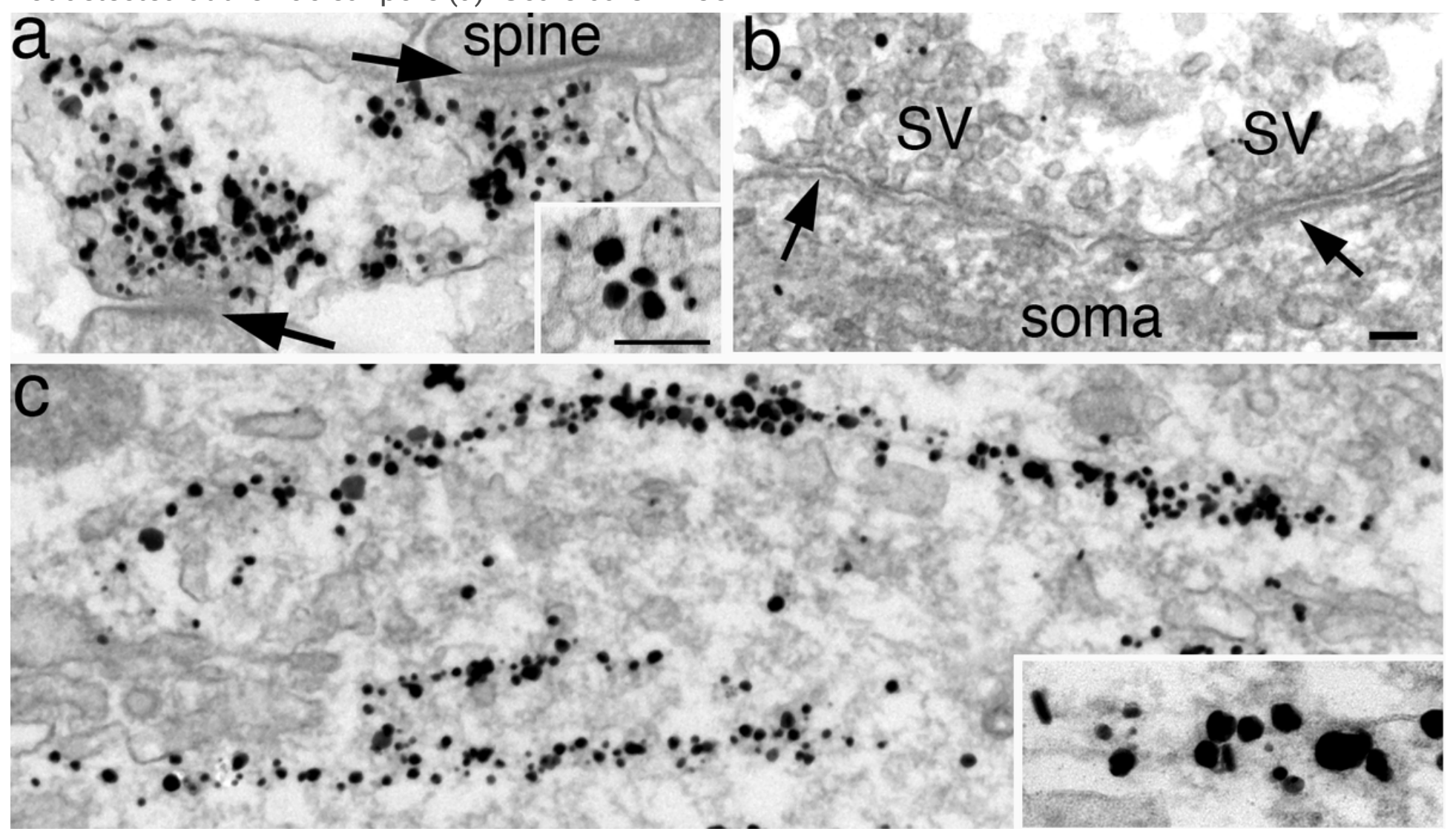

Figure 16

Dissociated hippocampal cultures labeled with a VGluT ab. Label was correctly localized to SVs of glutamatergic terminals ( $a$, arrows point to the characteristic PSDs of asymmetric synapses), but not to SVs of GABAergic terminals of symmetric synapses (b, arrows points to symmetric synapses). However, this antibody also cross reacted with a filamentous structure in the cytoplasm (c). Scale bars $=100 \mathrm{~nm}$. a-c share the same bar; insets of a \& c share the same bar. 

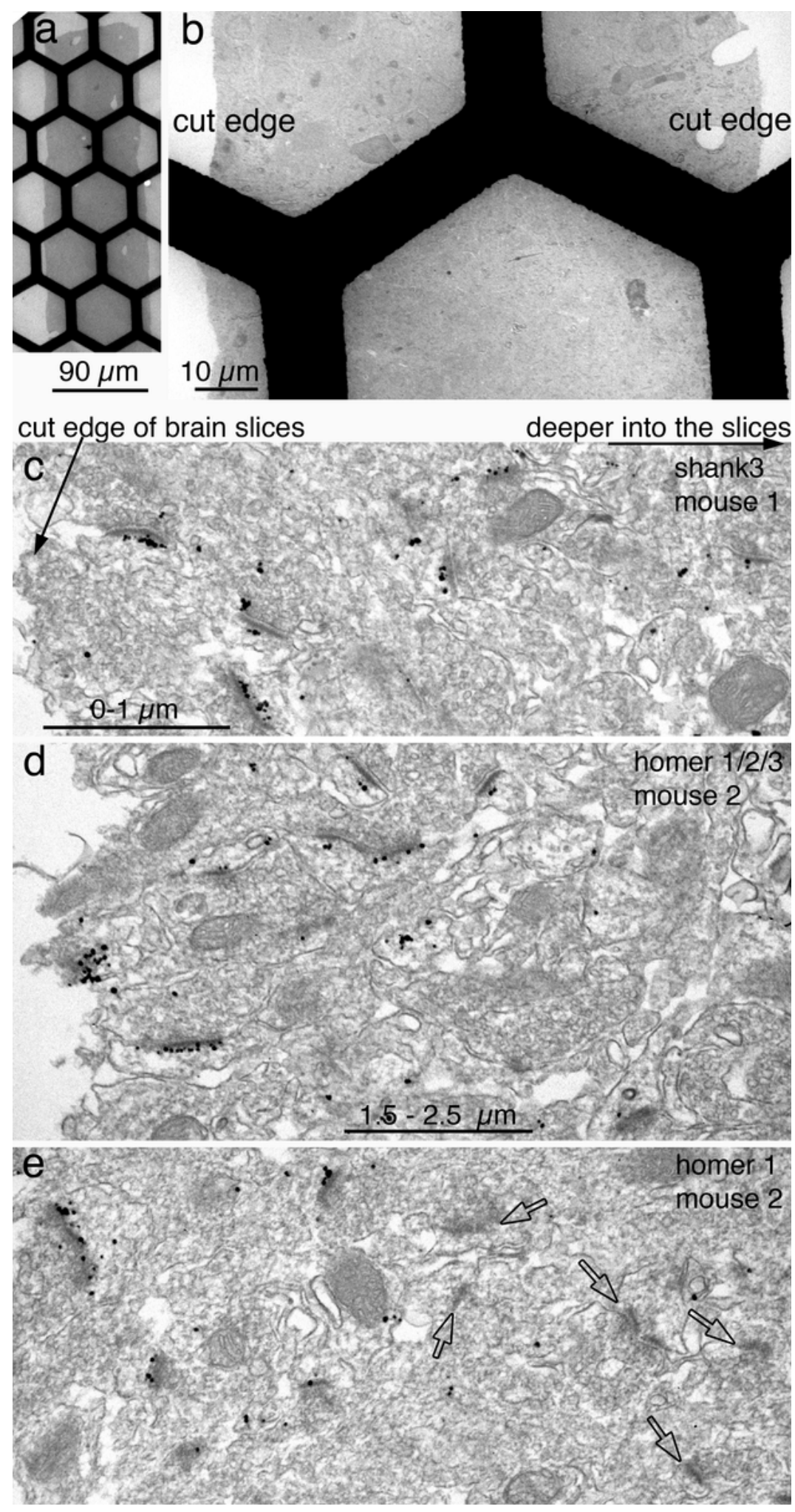

Figure 17

A cross-sectioned $90 \mu \mathrm{m}$ thick brain slice is collected on a 400-mesh hexagonal grid (a), and the two cut edges of the slice are enlarged in (b). Perfusion-fixed brain slices labeled with shank 3 (c), homer 1/2/3 (d), and homer 1 (e), three different antibodies against these PSD scaffold proteins [14, 23]. Labeling density decreases from the cut edges of the slices on left toward the deeper tissue of the slices on right (c-e). The penetration gradient is similar between $c$ and $d$ where two antibodies were used on slices from 
two different mice. However, depth of penetration for the third antibody (e) appeared to be shallower, even though the slice was from the same animal as in (d). Many PSDs were unlabeled (open arrows in e) beyond $2 \mu \mathrm{m}$ deep from the cut edge. Scale bar $=1 \mu \mathrm{m}$.

\section{CaMKII}

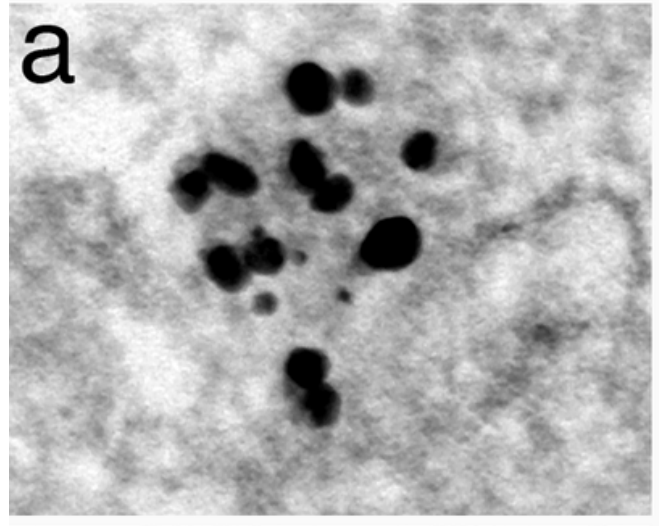

\section{d}

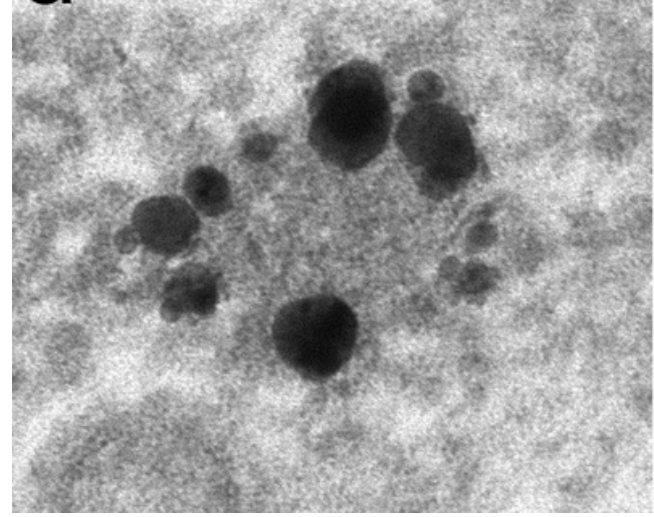

chromogranin A
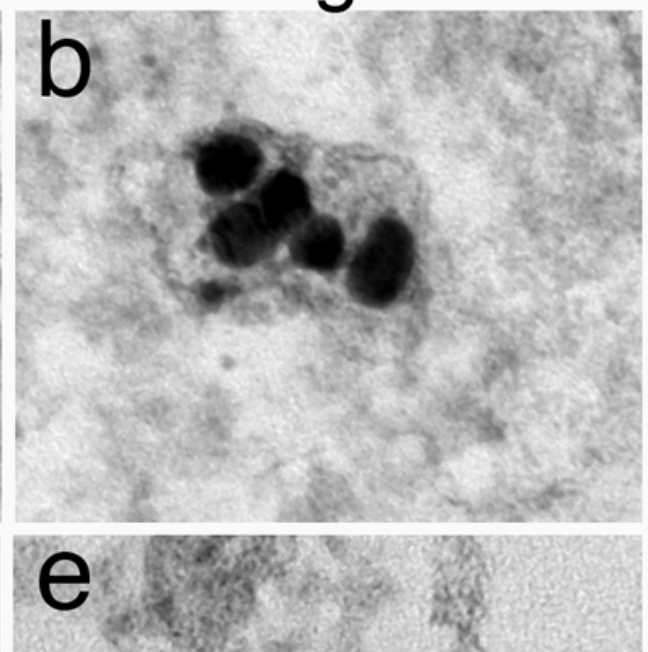

non-specific
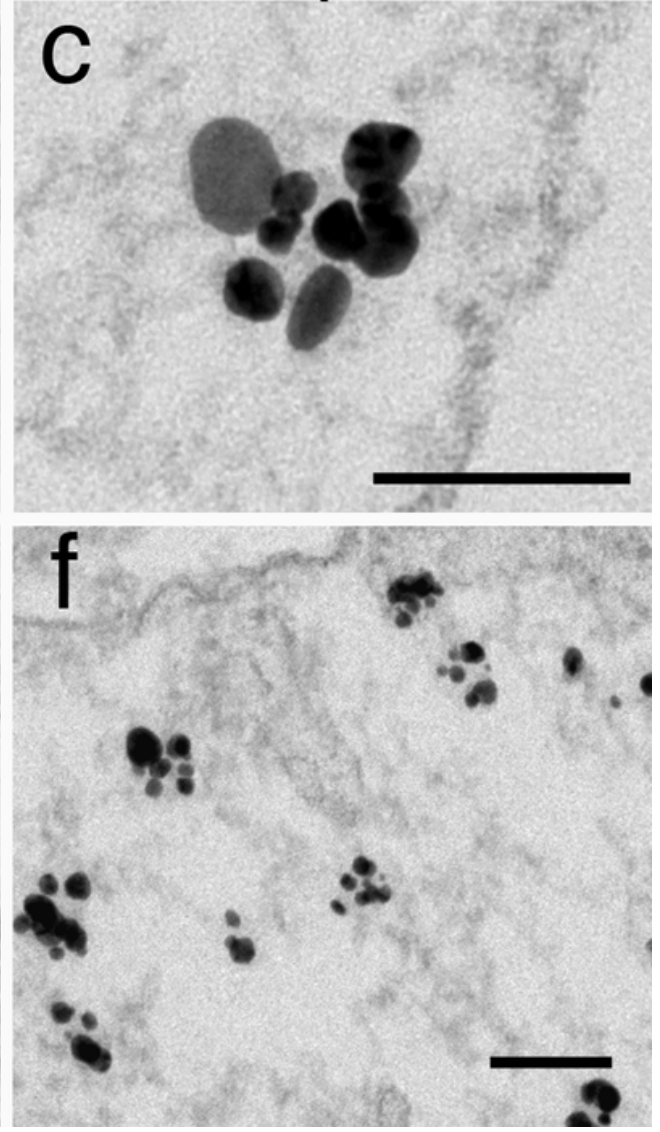

\section{Figure 18}

Dissociated hippocampal cultures labeled with CaMKII (a, d), chromogranin A (b, e) and two non-specific antibodies $(c, f)$. The clusters of signals in the left two colums were legitimate multiple signals of the aggregated proteins. The two primary antibodies used in $(c, f)$ turned out to be non-specific but these images serve the purpose of illustrating clumping of the secondary antibody, and these clusters should not be counted as multiple signals. Scale bars $=100 \mathrm{~nm}$.

\section{Supplementary Files}

This is a list of supplementary files associated with this preprint. Click to download.

- AdditionalFile1.pdf

- AdditionalFile2.pdf

- AdditionalFile3.pdf

- AdditionalFile4.pdf 